\title{
Métodos Bayesianos em Confiabilidade de Software
}

\author{
JuAn Esteban Ramírez Cid
}

Tese apresentada ao Instituto de Matemáticas

e Estatística da Universidade de São Paulo, para obtenção do grau de Doutor em Estatística.

Área de Concentração: Estatística

Orientador: Prof. Dr. JoRge Alberto ACHCAR

Suporte financeiro: $\mathrm{CNPq}$

Dezembro de 1999. 


\section{Métodos Bayesianos em Confiabilidade de Software}

Este exemplar corresponde à redação final devidamente corrigida e defendida por Juan Esteban Ramírez Cid e aprovada pela comissão julgadora.

São Paulo, 16 de Dezembro de 1999.

Banca examinadora:

- Prof. Dr. Jorge Alberto Achcar (Presidente)

- Prof. Dr. Heleno Bolfarine

- Prof. Dr. José Galvão Leite

- Prof. Dr. Francisco Louzada Neto

- Prof. Dr. Hélio Migon 


\section{Dedicatória}

A Deus.

A todos meus ascendentes, começando por Adão e Eva até meus pais Raúl e Efigenia. A meus descendentes, Esteban e Agustina. 


\section{Resumo}

Nesta tese introduzimos novos modelos para confiabilidade de software assumindo processos de Poisson não-homogêneos. Analises Bayesiana para os modelos propostos são consideradas usando métodos MCMC (Monte Carlo em Cadeias de Markov). Em geral, dados de confiabilidade de software envolvem opiniões de especialistas que podem ser incorporadas como distribuições a priori informativas.

Consideramos superposição de processos de Poisson não-homogêneos independentes com e sem introdução de covariáveis, superposição de processos de Poisson não-homogêneos assumindo dependência e uso de processo de Poisson não-homogêneo com função intensidade Weibull-exponenciada como alternativa ao uso de uma superposição de processos não-homogêneos.

Usamos métodos Bayesianos para discriminar diferentes modelos para dados de confiabilidade de software. 
Agradecimentos ao vivo. 


\section{ABSTRACT}

In this dissertation, we introduce new models for software reliability data assuming non-homogeneous Poisson processes. Bayesian analysis are considered for the proposed models using MCMC (Markov Chain Monte Carlo) methods. Usually, software reliability data have expert opinion which could be incorporated as informative prior distributions.

We consider superposition of independent non-homogeneous Poisson processes assuming and not assuming covariates, superposition of dependent non-homogeneous Poisson processes, and the use of non-homogeneous Poisson processes with a exponentiatedWeibull form for the intensity function.

We use Bayesian methods for discrimination of the proposed models for software reliability data. 


\section{Sumário}

1 Introdução

1.1 Motivação . . . . . . . . . . . . . . . . . . . . . 1

1.2 Modelos Básicos . . . . . . . . . . . . . . . . . . . . 2

1.2.1 Modelo de Jelinsky e Moranda . . . . . . . . . . . . . . . . . . . . 2

1.2.2 Modelo de Moranda . . . . . . . . . . . . . . . . . . 3

1.2.3 Modelo de Moranda generalizado . . . . . . . . . . . . . . . 3

1.2.4 Modelo de Goel e Okumoto . . . . . . . . . . . . . . . . . . 4

1.2.5 Modelo de Schick e Wolverton . . . . . . . . . . . . . . 4

1.2.6 Modelo de Littlewood e Verall . . . . . . . . . . . . . . . 5

1.2.7 Modelos baseados em processos PPNH. . . . . . . . . . . 5

1.3 Teoria unificada . . . . . . . . . . . . . . . . . 6

1.4 Função de verossimilhança . . . . . . . . . . . . . . . . 9

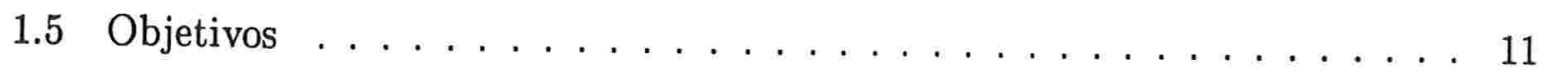

2 Confiabilidade de software considerando a superposição de processos PPNH na presença 
de uma covariada

2.1 Introdução . . . . . . . . . . . . . . . . . . . . . 16

2.2 Inferência Bayesiana para a superposição de modelos . . . . . . . . . . . . . . . . . . . . 18

2.3 Modelo 1: Superposição dos processos Musa e Okumoto com o processo Exponencial . . . . . . . . . . . . . . . . . . 20

2.4 Superposição de modelos na presença de uma covariada. . . . . . . . . . . . 22

2.4.1 Influência da covariada nas probabilidades de incidência. . . . . . . 22

2.4.2 Influência da covariada nas probabilidades de incidência e na função intensidade.

2.5 Algumas considerações relativas às estimativas de Bayes e à seleção de modelos

3 Uso da distribuição Weibull-

exponenciada para modelar funções

intensidade na forma de banheira

3.1 Introdução . . . . . . . . . . . . . . . . . . . 32

3.2 Um processo do tipo PPNH-II assumindo funções intensidades Weibull-exponenciada . . . . . . . . . . . . . . . . 34

3.3 Superposição de processos PPNH considerando a introdução de probabilidade de incidência . . . . . . . . . . . . . . . 36

3.4 Inferência Bayesiana e escolha do modelo . . . . . . . . . . . . . . 39

4 Superposição de processos PPNH dependentes 
4.1 Introdução . . . . . . . . . . . . . . . . . . . 40

4.2 Função de verossimilhança $\ldots \ldots \ldots \ldots \ldots$. . . . . . . . . 40

4.3 Inferência Bayesiana $\ldots \ldots \ldots \ldots \ldots \ldots \ldots \ldots \ldots$

4.3.1 Estimação da função de confiabilidade $\ldots \ldots \ldots$. . . . . . . 47

4.4 Exemplo $\ldots \ldots \ldots \ldots \ldots \ldots \ldots \ldots \ldots \ldots \ldots \ldots$

4.5 Modelagem Alternativa $\ldots \ldots \ldots \ldots \ldots \ldots$

5 Discriminação Bayesiana de modelos 53

5.1 Estimativa da verossimilhança marginal usando amostragem por importância $\ldots \ldots \ldots \ldots$. . . . . . . . 53

5.2 Fator de Bayes $\ldots \ldots \ldots \ldots \ldots \ldots \ldots \ldots \ldots$

6 Aplicações numéricas $\quad 58$

6.1 Exemplo para o Capítulo $2 \ldots \ldots \ldots \ldots \ldots \ldots$

6.1.1 Modelo 1: Superposição dos processos Musa e Okumoto com o processo Exponencial . . . . . . . . . . . . . . . . . . . . . 59

6.1.2 Modelo 2: Superposição dos processos Musa e Okumoto com o processo Exponencial incluindo uma covariada que afeta somente as probabilidades de incidência. . . . . . . . . . . . 60

6.1.3 Modelo 3: Superposição dos processos Musa e Okumoto com o processo Exponencial incluindo uma covariada que influencia as probabilidades de incidência e as funções intensidades. . . . . . . . . . 61

6.2 Exemplo para o Capítulo $3 \ldots \ldots \ldots 4 \ldots \ldots \ldots$

6.3 Exemplo para o Capítulo $4 \ldots \ldots \ldots \ldots \ldots$. . . . . . . . . 71 
A Processos de Poisson

A.1 Definições básicas . . . . . . . . . . . . . . . . 77

A.2 Processos de Poisson $\ldots \ldots \ldots \ldots \ldots \ldots \ldots$

B A distribuição de Hermite $\quad 81$

B.1 Definições . . . . . . . . . . . . . . . . . . . . . . . 81

B.2 Propriedades dos polinomios de Hermite, $H_{n}(x) \ldots \ldots \ldots \ldots$

B.3 Propriedades dos polinomios de Hermite modificados, $H_{n}^{*}(x) \ldots \ldots \ldots \ldots \ldots \ldots \ldots \ldots$

B.4 Cálculo da f.d.p. da distribuição de Hermite . . . . . . . . . . . . . 83

$\begin{array}{ll}\text { C A distribuição de Poisson Bivariada } & 85\end{array}$

D Geração de processos de Poisson 


\section{Capítulo 1}

\section{Introdução}

\subsection{Motivação}

O funcionamento de uma indústria, o atendimento de um banco e a modelagem econômica de um governo são somente alguns exemplos do dia a dia de um país, onde os sistemas computacionais dependem do sucesso de softwares envolvidos. Portanto é necessário estudar a qualidade do software usado, onde a confiabilidade de software tornase de extrema importância.

Um programador ou um engenheiro de software precisam avaliar e controlar bugs e falhas quando estão construindo e testando um novo programa. Esses bugs ou falhas devem ser detectados e corrigidos numa fase de testes.

Agora vamos introduzir um pouco mais da linguagem de software. Um bug ou erro é uma parte de um software que causa ou pode causar falha no funcionamento dele. O conceito de falha de um software é o mesmo de controle de qualidade: Uma falha de um software é produzida quando acontece um desvio na conduta do sistema. Uma definição de confiabilidade do software, que inclui uma idéia estocástica é devida a Musa e Okumoto, 1984 : A confiabilidade de software é a probabilidade de um programa computacional 
estar livre de erros de operação durante um período específico de tempo $(0, t]$. Neste caso, $t$ é chamado de período de teste.

Para modelagem estatística de dados de confiabilidade de software, a literatura apresenta muitos modelos probabilísticos e várias abordagens: as mais utilizadas são as estratégias de modelagem paramétrica para os tempos entre falhas e as estratégias de contagem de falhas num dado período de tempo por um processo de Poisson homogêneo (PPH) ou por um processo de Poisson não-homogêneo (PPNH), (veja Apêndice A).

A seguir, apresentamos alguns modelos muito usados na modelagem de dados de confiabilidade de software.

\subsection{Modelos Básicos}

\subsubsection{Modelo de Jelinsky e Moranda}

Este modelo foi proposto por Jelinsky e Moranda em 1972. Suponhamos que no começo do teste existem $N$ erros e que cada vez que o software falha um erro é corrigido e portanto o número de bugs decresce uma unidade e que cada um destes $N$ erros tem igual probabilidade de fazer que o sistema falhe. Também suponhamos que os tempos entre falhas são independentes e que a taxa de falhas é proporcional ao número de erros do sistema. Assim, a taxa de falhas depois que o $(i-1)$-ésimo erro apareça, pode ser expressa por

$$
\lambda_{i}=\lambda(N-i+1),
$$

onde $\lambda$ é a constante de proporcionalidade.

A função de densidade de probabilidade (f.d.p.), para o intervalo entre a (i-1)-ésima falha e a $i$-ésima falha é dada por:

$$
f\left(t_{i} \mid \lambda_{i}\right)=\lambda_{i} e^{-\lambda_{i} t_{i}},
$$


onde $\lambda_{i}$ é dado por (1.1) para $t_{i}>0$.

Uma das principais críticas que são feitas a este modelo é que as suposições sobre os erros podem não ser realistas, pois se supõe uma melhoria sempre crescente, isto é, cada vez que um erro é descoberto e corrigido não podemos ter novos erros introduzidos no programa.

\subsubsection{Modelo de Moranda}

Este modelo foi introduzido por Moranda em 1975. Consideramos um processo PPH para descrevermos o número de falhas em cada período sucessivo de tempo.

A taxa de falhas é da forma

$$
\lambda_{i}=\lambda_{a} k^{i}=\lambda k^{i-1}
$$

onde $\lambda=\lambda_{a} k$.

Padronizamos de tal forma que os períodos de tempo sejam do mesmo comprimento, (unitário). Assim, o número de falhas tem distribuição de Poisson dada por:

$$
P\left[X=m_{i}\right]=\frac{e^{-\lambda_{i}} \lambda_{i}^{m_{i}}}{m_{i} !}, \quad m_{i}=0,1,2, \cdots,
$$

onde $\lambda_{i}$ é dado em (1.3).

\subsubsection{Modelo de Moranda generalizado}

Uma generalização do modelo de Moranda é introduzida por Achcar, 1995, considerando a introdução de um parâmetro adicional.

A taxa de falhas é da forma:

$$
\lambda_{i}=\lambda_{a} k_{1}^{i k_{2}} \quad i=1,2, \cdots,
$$


onde $0<k_{1}<1, \quad-\infty<k_{2}<\infty$.

A introdução do parâmetro $k_{2}$ faz com que a taxa de falhas seja mais flexível, pois de acordo com os diferentes valores que pode tomar será monótona constante, decrescente ou crescente.

\subsubsection{Modelo de Goel e Okumoto}

Introduzido por Goel e Okumoto ,1979. Este modelo é similar ao modelo de Jelinsky e Moranda, mas assume que existe uma probabilidade $p \in[0,1]$ de consertar um bug quando este é encontrado.

A taxa de falhas é da forma:

$$
\lambda_{i}=\lambda[N-(i-1) p]
$$

Observamos que quando $\mathrm{p}=1$, temos o modelo de Jelinsky e Moranda da subseção 1.2.1.

\subsubsection{Modelo de Schick e Wolverton}

Este modelo foi introduzido em 1978. Supondo que a taxa de falhas é proporcional ao número de bugs que permanecem no sistema e ao tempo transcorrido desde a última falha. Assim, a taxa de falhas é da forma:

$$
\lambda_{i}=\lambda(N-i+1) t .
$$




\subsubsection{Modelo de Littlewood e Verall}

Este modelo foi introduzido por Littlewood e Verall, 1973. A idéia dos autores é relaxar a suposição de reparo perfeito do modelo de Jelinsky e Moranda da subseção 1.2.1.

Supor que o i-ésimo tempo entre falhas tem uma f.d.p. exponencial com taxa de falhas $\lambda_{i}$ tal que,

$$
P\left[\lambda_{i+1}<\lambda\right] \geq P\left[\lambda_{i}<\lambda\right] \quad i=1,2, \cdots ; \quad \lambda \geq 0 .
$$

Assim, Littlewood e Verall consideram uma distribuição gama para $\lambda_{i}$ com parâmetro de escala $\psi(i)$, onde $\psi(i)$ é uma função monótona crescente de $i$, ou seja:

$$
\pi\left(\lambda_{i} \mid \alpha, \psi(i)\right)=\frac{[\psi(i)]^{\alpha}}{\Gamma(\alpha)} \lambda_{i}^{\alpha-1} e^{-\psi(i) \lambda_{i}}
$$

A função $\psi(i)$ descreve a qualidade do programador e a tarefa de programar. Mazzuchi e Soyer, 1988, assumem que a função $\psi(i)$ é polinomial, ou seja:

$$
\psi_{i}=\beta_{0}+\beta_{1} i+\cdots+\beta_{k} i^{k}
$$

e também supõem que é uma função não decrescente e portanto que o tempo entre as falhas aumenta ao serem removidos os bugs.

\subsubsection{Modelos baseados em processos PPNH.}

Antes que fosse desenvolvida a teoria unificada (veja, Seção 1.3), foram usados alguns modelos que envolviam processos PPNH, (veja, Apêndice A), para modelar as falhas, como por exemplo, 
Modelo de Duane e Crow, 1966.

$$
\lambda(t)=\alpha \beta t^{\beta-1} \quad t \geq 0, \quad \alpha>0, \quad \beta>0 .
$$

Modelo de Goel e Okumoto, 1979.

$$
\lambda(t)=\alpha \beta e^{\beta t} \quad t \geq 0, \quad \alpha>0, \quad \beta>0 .
$$

Modelo de Musa e Okumoto, 1984.

$$
\lambda(t)=\frac{\alpha}{\beta+t} \quad t \geq 0, \quad \alpha>0, \quad \beta>0
$$

\subsection{Teoria unificada}

$\mathrm{Na}$ maioria dos exemplos dos modelos mais freqüentemente usados em confiabilidade de software , podemos observar que em cada um deles é utilizada uma das duas estratégias seguintes:

(i) Estratégia de tipo I : Aquela que modela os tempos entre falhas sucessivas do software,$\left(t_{i-1}, t_{i}\right)$.

(ii) Estratégia de tipo II : Aquela que modela o número de falhas do software num período de tempo determinado $(0, t]$.

No primeiro caso é comum usar uma distribuição exponencial, enquanto que no último caso é usado um processo pontual para contar o número de falhas. As alternativas 
mais comuns são o uso de um processo PPH ou de um processo PPNH. Neste caso usamos a seguinte notação:

$M(t)=$ Número acumulado de falhas em $(0, t]$.

$m(t)=E[M(t)]$ é chamada de função de valor médio.

A função intensidade $\lambda$ é tal que:

$$
m(t)=\int_{0}^{t} \lambda(u) d u
$$

Portanto,

$$
m^{\prime}(t)=\lambda(t)
$$

A flexibilidade do modelo está relacionada com as diferentes formas que a função intensidade pode assumir.

Observamos que se a função intensidade é constante, então o processo PPNH se reduz ao caso de processo PPH. Isto é: $\lambda(u)=\lambda$ para todo $u$. Assim, $m(t)=\lambda t$.

$\mathrm{Na}$ literatura, observamos que vários autores tem trabalhado no sentido de unificar os vários modelos propostos por especialistas em software .

Langberg e Singpurwalla, 1985, consideram os modelos G.O. ( Goel e Okumoto) e L.V. ( Littlewood e Verall) como generalização do modelo J.M. (Jelinsky e Moranda).

Outra generalização é proposta por Miller, 1986, considerando os tempos entre falhas como variáveis aleatórias com distribuições exponenciais, mas não idênticas.

Uma unificação da maioria dos modelos existentes para modelar dados de confiabilidade do software, é dada por Yang, 1994.

Yang, 1994, classifica os modelos de tempos de falha em duas classes:

1. Modelos GOS ou modelos de estatísticas de ordem generalizados.

2. Modelos RVS ou modelos de estatísticas de valores observados. 
Os modelos GOS assumem que existe um número desconhecido de falhas $N$ no começo do processo de debugging ou teste e modela as épocas observadas de falha como as primeiras $n$ estatísticas de ordem tomadas das $N$ observações i.i.d. com f.d.p. com suporte em $\mathcal{R}+$. Assumimos que neste modelo ao repararmos alguma falha não são introduzidas novas falhas .

Os modelos do tipo RVS permitem a introdução de novas falhas após o reparo de uma falha. Os modelos RVS assumem que os tempos de falhas são estatísticas observadas i.i.d. com f.d.p. com suporte em $\mathcal{R}+$.

Sob o ponto de vista dos processos de contagem, a teoria unificada proposta por Yang considera a classificação dos modelos de acordo com o número de falhas: finito ou infinito. Tanto para os modelos GOS quanto para os modelos RVS, $M(t)$ está relacionado com um processo PPNH. No caso GOS estamos supondo que $N$ tem distribuição de Poisson com média $\theta$. Assim, temos que $M(t)$ é um processo PPNH com função de valor médio

$$
m(t)=\theta F(t)
$$

onde $F$ é a função de distribuição acumulada (F.D.A. ) com f.d.p., f. Obviamente, neste caso,

$$
\lim _{t \rightarrow \infty} m(t)<\infty
$$

Yang chama este tipo de modelos de processos PPNH-I.

Os modelos para os quais ocorre que

$$
\lim _{t \rightarrow \infty} m(t)=\infty
$$

são chamados de processos PPNH-II.

Dado um modelo RVS derivado a partir de amostras i.i.d. de $f$, podemos provar 
que o correspondente processo $M(t)$ é do tipo PPNH-II com função de valor médio

$$
m(t)=-\log (1-F(t))
$$

A implicação inversa pode ser estabelecida da seguinte forma: tempos de falha de um processo PPNH-II com função intensidade $\lambda(t)$ correspondem a um modelo RVS derivado de observações i.i.d. com f.d.p. comum dada por:

$$
f(t)=\lambda(t) e^{-\int_{0}^{t} \lambda(u) d u} .
$$

\subsection{Função de verossimilhança}

Para o modelo de tempo truncado o conjunto de dados pode ser escrito na forma seguinte:

$$
\mathcal{D}_{t}=\left\{x_{1}, \cdots, x_{n}\right\},
$$

onde $x_{1}, \cdots, x_{n}$ são os tempos onde ocorrem as $n$ falhas e $t$ é o tempo de truncamento.

No caso do modelo de falhas truncado, ou seja onde observamos até a ocorrência da n-ésima falha o conjunto de dados é denotado por:

$$
\mathcal{D}_{x_{n}}=\left\{x_{1}, \cdots, x_{n}\right\}
$$

Na sua tese, Yang, 1994, apresenta duas op̧̧ões para obter a verossimilhança : Método 1.

Dado o modelo de tempo truncado, o número de falhas observadas em $(0, t]$ é uma variável aleatória denotada por $n$.

Dado $M(t)=n$, os tempos

$$
x_{1}<x_{2}<\cdots<x_{n}<t
$$


tem a mesma distribuição das estatísticas de ordem de v.a.i.i.d com F.D.A. comum dada por,

$$
F(\omega)=\frac{m(\omega)}{m(t)} \quad 0 \leq \omega \leq t
$$

Assim, a função de verossimilhança é dada por,

$$
\begin{aligned}
L\left(\theta, \underline{\beta} \mid \mathcal{D}_{t}\right) & =P\left[\mathcal{D}_{t} \mid M(t)=n\right] P[M(t)=n] \\
& =n !\left[\prod_{i=1}^{n} \frac{\lambda\left(x_{i}\right)}{m(t)}\right] \frac{e^{-m(t)}[m(t)]^{n}}{n !} \\
& =\left[\prod_{i=1}^{n} \lambda\left(x_{i}\right)\right] e^{-m(t)},
\end{aligned}
$$

onde $\underline{\beta}$ é o vetor de parâmetros.

\section{Método 2.}

A sequência de tempos

$$
x_{1}<x_{2}<\cdots,
$$

é um processo PPNH, e a sequência

$$
m\left(x_{1}\right)<m\left(x_{2}\right)<\cdots,
$$

é um processo PPH com taxa 1, logo os tempos entre as chegadas são distribuídos exponencialmente com taxa 1 .

Assim,

$$
P\left[m\left(X_{n}\right) \geq m\left(X_{n-1}+t\right)\right]=P\left[X_{n}-X_{n-1}>t\right] .
$$

Logo,

$$
\begin{aligned}
P\left[X_{n}-X_{n-1}>t \mid X_{1}, \cdots, X_{n-1}\right] & =P\left[m\left(X_{n}\left|\geq m\left(X_{n-1}+t\right)\right| X_{1}, \cdots, X_{n-1}\right]\right. \\
& =\exp \left\{-\left(m\left(X_{n-1}+t\right)-m\left(X_{n-1}\right)\right)\right\} \\
& =P\left[X_{n}-X_{n-1}>t \mid X_{n-1}\right] .
\end{aligned}
$$


Além disso,

$$
\begin{aligned}
f_{X_{1}}\left(x_{1}\right) & =\lambda\left(x_{1}\right) \exp \left\{-m\left(x_{1}\right)\right\} \\
f_{X_{n} \mid X_{n-1}}\left(x_{n} \mid x_{n-1}\right) & =\lambda\left(x_{n}\right) \exp \left\{-\left(m\left(x_{n}\right)-m\left(x_{n-1}\right)\right)\right\} .
\end{aligned}
$$

Portanto, a função de verossimilhança para o modelo de tempo truncado é:

$$
\begin{aligned}
L\left(\theta, \underline{\beta} \mid \mathcal{D}_{t}\right) & =f\left(x_{1}, x_{2}, \cdots, x_{n} \mid t\right) \\
& =f_{X_{1}}\left(x_{1}\right) f_{X_{2} \mid X_{1}}\left(x_{2} \mid x_{1}\right) \cdots f_{X_{n} \mid X_{n-1}}\left(x_{n} \mid x_{n-1}\right) P\left[X_{n+1}>t \mid x_{n}\right],
\end{aligned}
$$

e daqui obtemos a mesma expressão (1.15).

No caso do modelo de falhas truncado obtemos uma expressão análoga a (1.15), que é dada por:

$$
L\left(\theta, \underline{\beta} \mid \mathcal{D}_{x_{n}}\right)=\left[\prod_{i=1}^{n} \lambda\left(x_{i}\right)\right] e^{-m\left(x_{n}\right)}
$$

\subsection{Objetivos}

Kuo e Yang, 1996, introduzem uma análise Bayesiana para modelos de processos PPNH, considerando o amostrador de Gibbs, (veja, por exemplo, Gelfand e Smith, 1990), para obter sumários de interesse. Eles consideram diferentes modelos sob processos PPNH assumindo processos GOS ou RVS. Achcar, Dey e Niverthi, 1998, consideram o uso de um supermodelo, a distribuição gama generalizada para modelar a função de valor médio (1.10) , o que generaliza vários resultados obtidos por, Kuo e Yang (1996).

Neste trabalho, consideramos o uso de amostrador de Gibbs com algoritmos MetropolisHastings (veja, por exemplo, Metropolis et al., 1953 ou Chib e Greenberg, 1995 ou Smith e Roberts, 1993, para casos especiais de superposição de processos PPNH). 
O uso de superposição de processos PPNH permite uma grande flexibilidade na modelagem da função de valor médio $m(t)$ incluindo formas crescentes, decrecentes ou em forma de banheira ( bathtub ). No capítulo 2 introduzimos o uso de métodos Bayesianos para superposição de processos PPNH na presença de covariáveis.

No capítulo 3, consideramos o uso de Métodos de Monte Carlo em Cadeias de Markov (MCMC) para obtermos inferências Bayesianas para uma classe especial de modelos com processos PPNH-II: O uso da forma Weibull-exponenciada (veja por exemplo, Mudholkar et al., 1995 ou Cancho, V.G., Bolfarine, H., e Achcar, J.A., 1999.) para a função intensidade $\lambda(t)$. Com esta escolha para $\lambda(t)$, podemos ter funções intensidades não-monótonas. Além disso, comparamos essa modelagem com a modelagem baseada na superposição de processos PPNH .

Observamos que alguns modelos dados nos capítulos 2 e 3 são misturas de funções intensidades. Por exemplo, temos os modelos (2.14) e (3.7), da forma geral,

$$
\lambda(t \mid \underline{\theta}, \underline{\beta})=\sum_{j=1}^{J} \theta_{j} \lambda_{j}\left(t \mid \underline{\beta_{j}}\right),
$$

e os casos particulares (2.16) e (3.8) respectivamente, onde $J=2$. Neste caso, surge um problema de não-identificabilidade, (veja, Gelman et al.,1995, ou Titterington et al., 1985). Isto ocorre devido ao fato de que a função intensidade $\lambda$ não muda se permutamos os índices das funções intensidades componentes. Porém, este problema é resolvido especificando a ordem das proporções $\theta$, por exemplo nos casos gerais especificando no modelo que

$$
\theta_{1} \leq \theta_{2} \leq \cdots \leq \theta_{J}
$$

e nos casos particulares especificando que $\theta_{1} \leq 0.5$

Os tempos que pretendemos modelar usando processos PPNH podem ser correlacionados. Um exemplo real, na área médica, é dado em Aslanidou H.,et al., 1998. Na área de modelagem de software, quando existe correlação entre dois processos de Poisson não-homogêneos para modelar dados de software, introduzimos processos PPNH usando 
uma distribuição de processos PPNH bivariada, e desenvolvemos a teoria necessária para estudar a superposição de dois processos PPNH dependentes. Mostramos como varia o coeficiente de correlação através do tempo e como varia a função intensidade comparada com o caso em que os processos são independentes, que resulta ser um caso particular desta modelagem. Fazemos este estudo considerando o uso de métodos Bayesianos com procedimentos MCMC para obter as inferências de interesse. Este é o conteúdo do capítulo 4. Este capítulo é o resultado de uma pesquisa desenvolvida em conjunto com o Prof. Dr. Josemar Rodrigues.

Todos os exemplos incluídos nesta tese para ilustrar a teoria são desenvolvidos no capítulo 6. Devido à impossibilidade de dispor de dados reais, todos os dados foram gerados usando os algoritmos apresentados no Apêndice D. Para verificar a convergência do procedimento MCMC usamos o método de Gelman e Rubin, (veja, Gelman et al., 1992). Encontramos que foi suficiente gerar 10 cadeias separadas, cada uma com 1200 iterações, eliminando os primeiros 200 elementos de cada cadeia, e selecionando um elemento de cada 10, em cada cadeia. Assim, obtivemos uma amostra de tamanho 1000 para cada parâmetro. Somente num exemplo do capítulo 2 usamos 2200 iterações para conseguir uma amostra final de tamanho 2000.

Nas aplicações reais a escolha das distribuições a priori pelos pesquisadores, geralmente incorporam conhecimento de especialistas e informação dos engenheiros relativo ao software. Assim, por exemplo, Campodonico S. e Singpurwalla N.D., 1995, consideram a escolha das distribuições a priori para modelos baseados em processos PPNH, sustentada nas informações de um especialista, por exemplo, relativas ao número de módulos, ao perfil operacional, etc. A partir desta informação consideram uma distribuição a priori conjunta para a função de valor médio, $m(t)$, para diferentes valores de $t$.

Para discriminar os vários modelos propostos na análise de dados de confiabilidade de software, consideramos o fator de Bayes baseado na função de verossimilhança marginal. Maiores detalhes são dados no capítulo 5. 
Na seguinte página apresentamos um fluxograma para mostrar numa forma ordenada o trabalho efetuado para desenvolver esta tese em torno da superposição de processos PPNH. Dois casos gerais são considerados, de acordo com a suposição de independência das componentes ou não. No primeiro caso, chamamos de superposição simples o caso estudado por Yang, 1994. Todos os outros casos mostrados no fluxograma, correspondem ao trabalho desenvolvido na tese. 


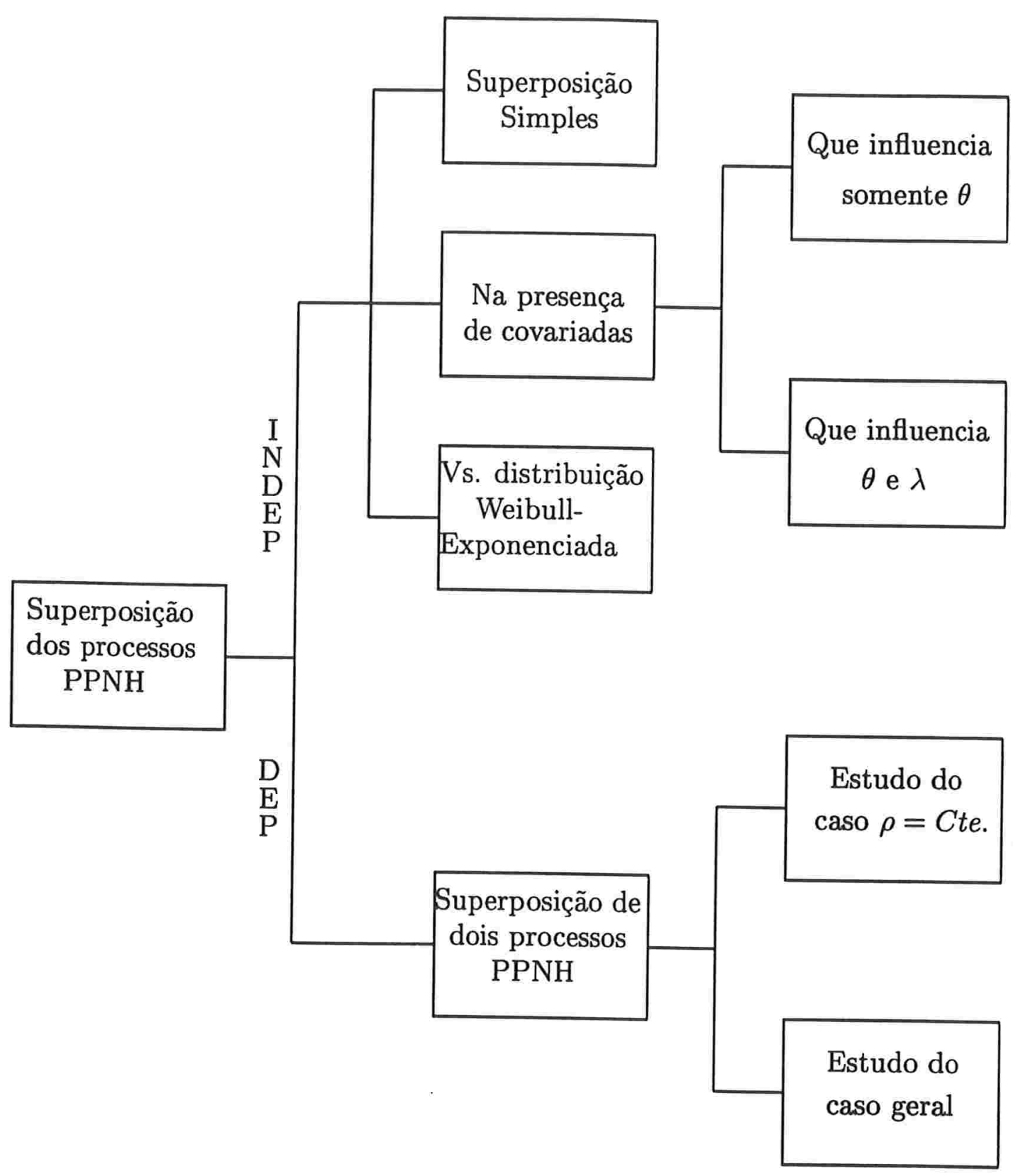

Figura 1.1. Diagrama de fluxo para visualisar o trabalho desenvolvido com a superposição de processos PPNH. 


\section{Capítulo 2}

\section{Confiabilidade de software}

\section{considerando a superposição de} processos PPNH na presença de uma covariada

\subsection{Introdução}

Consideramos a modelagem do número de falhas de um software usando um processo pontual para contar o número de falhas (veja Musa, Ianino e Okumoto, 1987).

Seja $M(t)$ o número acumulado de falhas de software que são observadas durante o tempo $(0, t]$.

Suponhamos que $M(t)$ é modelado por um processo PPNH com função intensidade

$$
\lambda(t)=\frac{d E[M(t)]}{d t},
$$

a qual assumimos uma função monótona de $t$ (veja Musa e Okumoto, 1984 ou Cox e 
Lewis, 1966).

Uma alternativa à consideração de funções monótonas é pensar na superposição de vários processos PPNH independentes. Kuo e Yang, 1996, desenvolveram metodologias de inferência Bayesiana e seleção de modelos para a superposição de modelos independentes.

O conjunto de pontos de falhas de um processo de superposição é definido como a união dos conjuntos dos pontos de falha dos processos componentes (veja Fig.2.1), onde $P_{i}$ representa o conjunto de pontos de falhas do processo $i, \quad i=1,2$ e $P_{3}$ representa o conjunto de pontos de falha da superposição dos processos $P_{1}$ e $P_{2}$.

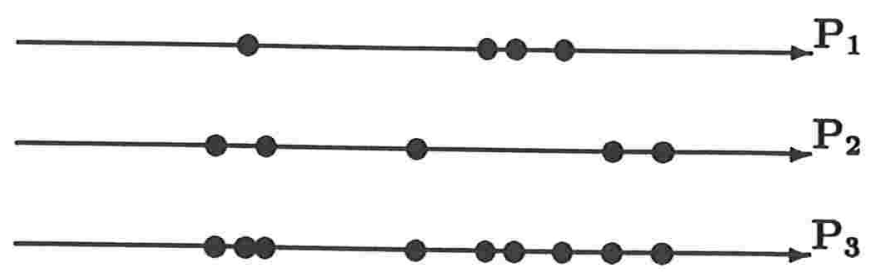

Figura 2.1. Ilustração do conjunto de pontos de falha da superposição de dois processos.

Seja $M_{j}(t)$ o processo PPNH que representa o número acumulado de falhas em $(0, t]$ para o j-ésimo processo componente com função intensidade $\lambda_{j}\left(t \mid \underline{\beta_{j}}\right)$, cuja forma assumimos conhecida, mas que inclui o vetor de parâmetros $\underline{\beta_{j}}$ desconhecido.

Assumimos também que os $M_{j}(t), \quad j=1, \cdots, J$ são mutuamente independentes.

Daí, podemos provar que o processo

$$
M(t)=\sum_{j=1}^{J} M_{j}(t)
$$


o qual conta o número total de falhas no intervalo $(0, t]$ é um processo PPNH com função intensidade,

$$
\lambda(t \mid \underline{\beta})=\sum_{j=1}^{J} \lambda_{j}\left(t \mid \underline{\beta_{j}}\right),
$$

onde $\underline{\beta}=\left(\underline{\beta_{1}}, \cdots, \underline{\beta_{J}}\right)$.

Uma das vantagens da superposição de processos PPNH é que podemos ter diferentes formas de função intensidade: funções de tipo banheira, polinômios com picos e vales e funções monótonas simples. Alguns casos especiais são os seguintes:

$$
\begin{aligned}
\text { (i) } \lambda(t) & =\alpha_{1} \beta_{1} t^{\alpha_{1}-1}+\alpha_{2} \beta_{2} t^{\alpha_{2}-1} \quad \text { (duas funções intensidades Weibull), } \\
\text { (ii) } \lambda(t) & =\frac{\alpha_{1}}{t+\beta_{1}}+\alpha_{2} t+\alpha_{3} \quad \text { (Gaver and Acar, 1979), } \\
\text { (iii) } \lambda(t) & =\beta_{0}+\beta_{1} t+\ldots+\beta_{m} t^{m} .
\end{aligned}
$$

Nas seções seguintes consideramos o uso do algoritmo de Gibbs (veja, Casella e George, 1992 ou Gelfand e Smith, 1990) e o algoritmo Metropolis-Hastings (veja, Chib e Greenberg, 1995 ou Smith e Roberts, 1993), para fazer uma abordagem Bayesiana da superposição de processos PPNH na presença de uma covariada.

\subsection{Inferência Bayesiana para a superposição de modelos}

Seja $\mathcal{D}_{t}$ o conjunto de dados, considerando o modelo de tempo truncado. A função de verossimilhança é dada por,

$$
L\left(\underline{\beta} \mid \mathcal{D}_{t}\right)=\left(\prod_{i=1}^{n} \lambda\left(t_{i}\right)\right) \exp (-m(t)),
$$


onde, $\underline{\beta}=\left(\underline{\beta_{1}}, \cdots, \underline{\beta_{J}}\right)$ e $m(t)=\int_{0}^{t} \lambda(u) d u,($ veja Cox e Lewis, 1966 uu Basawa e Rao, 1980). Para o modelo de falhas truncado, uma expressão similar pode ser usada com $t$ substituído por $t_{n}$.

Assumimos que as distribuições a priori para $\underline{\beta_{j}}, \quad \pi_{j}\left(\underline{\beta_{j}}\right) \quad j=1, \cdots, J$ são independentes.

A densidade a posteriori para $\underline{\beta}$ é dada por,

$$
\pi\left(\underline{\beta} \mid \mathcal{D}_{t}\right) \propto\left(\prod_{i=1}^{n} \sum_{j=1}^{J} \lambda_{j}\left(t_{i}\right)\right) \exp \left(-\sum_{j=1}^{J} m_{j}(t)\right) \prod_{j=1}^{J} \pi_{j}\left(\beta_{j}\right) .
$$

Para simplificar as distribuições condicionais que são necessárias para o uso do algoritmo de Gibbs, consideramos a técnica de Data augmentation (veja Tanner e Wong, 1987), mediante a inclusão de variáveis latentes.

Sejam $\underline{I_{i}}=\left(I_{i 1}, \cdots, I_{i J}\right), \quad i=1, \cdots, n$, onde $I_{i j}=1$ se a i-ésima falha é causada pelo j-ésimo tipo e $I_{i j}=0$ caso contrário. Observe que $\sum_{j=1}^{J} I_{i j}=1$ para $i=1, \cdots, n$.

Assumimos que a distribuição condicional para $\underline{I_{i}}$ dado $\underline{\beta}$ e $\mathcal{D}_{t}$ é uma distribuição multinomial com parâmetros 1 e probabilidades de celas $\left(p_{i 1}, \ldots, p_{i J}\right)$, onde

$$
p_{i j}=\frac{\lambda_{j}\left(t_{i}\right)}{\sum_{j=1}^{J} \lambda_{j}\left(t_{i}\right)} .
$$

Portanto, temos:

$$
\pi\left(\underline{I_{i}} \mid \underline{\beta}, \mathcal{D}_{t}\right) \propto \prod_{j=1}^{J} p_{i j}^{I_{i j}} \propto \prod_{j=1}^{J}\left[\frac{\lambda_{j}\left(t_{i}\right)}{\sum_{j=1}^{J} \lambda_{j}\left(t_{i}\right)}\right]^{I_{i j}} .
$$

Assim, a densidade a posteriori de $\underline{\beta}$ dado $I=\left(\underline{I_{1}}, \cdots, \underline{I_{n}}\right)^{T}$, ( uma matriz nxJ ), e $\mathcal{D}_{t}$ é dada por,

$$
\pi\left(\underline{\beta} \mid I, \mathcal{D}_{t}\right) \propto L\left(\underline{\beta} \mid \mathcal{D}_{t}\right)\left(\prod_{i=1}^{n} \pi\left(\underline{I_{i}} \mid \underline{\beta}, \mathcal{D}_{t}\right)\right)\left(\prod_{j=1}^{J} \pi_{j}\left(\underline{\beta_{j}}\right)\right) .
$$


Ou seja,

$$
\pi\left(\underline{\beta} \mid I, \mathcal{D}_{t}\right) \propto \prod_{j=1}^{J} \prod_{i: I_{i j}=1} \lambda_{j}\left(t_{i}\right) \prod_{j=1}^{J} \exp \left(-m_{j}(t)\right) \prod_{j=1}^{J} \pi_{j}\left(\underline{\beta_{j}}\right) .
$$

\subsection{Modelo 1: Superposição dos processos Musa e Okumoto com o processo Exponencial}

Este caso foi estudado por Yang, em 1996, sob a hipótese de independência e usando uma abordagem Bayesiana. As funções intensidades dos processos Musa e Okumoto com o processo exponencial são, respectivamente,

$$
\begin{aligned}
\lambda_{1}(t) & =\frac{\alpha}{\beta_{1}+t} \\
\lambda_{2}(t) & =\beta_{2} t .
\end{aligned}
$$

Logo, a função intensidade do processo superposição é dada por

$$
\lambda(t)=\frac{\alpha}{\beta_{1}+t}+\beta_{2} t
$$

e a função de valor médio do processo superposição é dada por,

$$
m(t)=\alpha \log \left(1+\frac{t}{\beta_{1}}\right)+\frac{\beta_{2} t^{2}}{2}
$$

Assumimos as seguintes distribuições a priori para $\alpha, \beta_{1}$ e $\beta_{2}$ :

$$
\begin{aligned}
& \alpha \sim \Gamma\left(a_{1}, b_{1}\right) ; \quad a_{1}, b_{1} \quad \text { conhecidas } \\
& \beta_{1} \sim \Gamma\left(a_{2}, b_{2}\right) ; \quad a_{2}, b_{2} \quad \text { conhecidas } \\
& \beta_{2} \sim \Gamma\left(a_{3}, b_{3}\right) ; \quad a_{3}, b_{3} \quad \text { conhecidas }
\end{aligned}
$$

onde, $\Gamma(a, b)$ denota a distribuição gama com média $a / b$ e variância $a / b^{2}$. Também assumimos independência entre os parâmetros $\alpha, \beta_{1}$ e $\beta_{2}$. 
Substituindo em (2.3) encontramos a função de verossimilhança

$$
L\left(\alpha, \beta_{1}, \beta_{2} \mid \mathcal{D}_{t}\right)=\left(\prod_{i=1}^{n}\left(\left(\frac{\alpha}{\beta_{1}+t_{i}}\right)+\beta_{2} t_{i}\right)\right) \exp \left(-\alpha \log \left(1+\frac{t_{n}}{\beta_{1}}\right)-\frac{\beta_{2} t_{n}^{2}}{2}\right) .
$$

Consideramos as variáveis latentes $I_{i j}=1 \quad i=1, \cdots, n$ e $j=1,2$ definidas na seção anterior.

Observamos que $I_{i 1}+I_{i 2}=1$. Logo, neste caso basta que geremos as variáveis $I_{i 1} \sim \operatorname{Bernoulli}\left(p_{i 1}\right)$ onde,

$$
p_{i 1}=\frac{\frac{\alpha}{\beta_{1}+t_{i}}}{\frac{\alpha}{\beta_{1}+t_{i}}+\beta_{2} t_{i}}=\frac{\alpha}{\alpha+\beta_{2}\left(\beta_{1}+t_{i}\right) t_{i}},
$$

ou seja, a f.d.p. de $I_{i 1}$ é

$$
\Pi\left(I_{i 1}\right)=p_{i 1}^{I_{i 1}}\left(1-p_{i 1}\right)^{1-I_{i 1}}
$$

Portanto,

$$
\Pi\left(I_{i 1}\right)=\frac{\alpha^{I_{i 1}}\left(\beta_{2}\left(\beta_{1}+t_{i}\right)\right)^{1-I_{i 1}}}{\alpha+\beta_{2}\left(\beta_{1}+t_{i}\right) t_{i}}
$$

Assim, a f.d.p. conjunta das variáveis latentes $I_{i 1}$ é,

$$
\prod_{i=1}^{n} \Pi\left(I_{i 1}\right) \propto \frac{\alpha^{\sum_{i=1}^{n} I_{i 1}} \beta_{2}^{n-\sum_{i=1}^{n} I_{i 1}} \prod_{i=1}^{n}\left(\left(\beta_{1}+t_{i}\right)\right)^{1-I_{i 1}}}{\prod_{i=1}^{n}\left(\alpha+\beta_{2}\left(\beta_{1}+t_{i}\right) t_{i}\right)} .
$$

Considerando as distribuições a priori dadas em (2.8) e substituindo as equações (2.9) e (2.11) na equação (2.5), obtemos a distribuição conjunta a posteriori para $\alpha, \beta_{1} \mathrm{e}$ $\beta_{2}$. Por motivos de simplicidade usaremos a notação $\underline{\beta_{1}}=\left(\alpha, \beta_{1}, \beta_{2}\right)$,

$$
\begin{gathered}
\pi\left(\underline{\beta_{1}} \mid I, \mathcal{D}_{t}\right) \propto \exp \left(-b_{2} \beta_{1}-\left(b_{1}+\log \left(1+\frac{t_{n}}{\beta_{1}}\right)\right) \alpha-\left(b_{3}+\frac{t_{n}^{2}}{2}\right) \beta_{2}\right) \\
\cdot \frac{\alpha^{a_{1}-1+\sum_{i=1}^{n} I_{i 1}} \beta_{1}^{a_{2}-1} \beta_{2}^{n+a_{3}-1-\sum_{i=1}^{n} I_{i 1}}}{\prod_{i: I_{i 1}=1}^{n}\left(\beta_{1}+t_{i}\right)} .
\end{gathered}
$$

As distribuições condicionais para o algoritmo de Gibbs são dadas por: 
(i) Construir I dado $\beta$ e $\mathcal{D}_{t}$ gerando variáveis aleatórias independentes $I_{i 1}$ com distribuição Bernoulli com parâmetros $p_{i 1}$ dados por (2.10).

(ii)

$$
\pi\left(\alpha \mid \beta_{1}, \beta_{2}, I, \mathcal{D}_{t}\right) \sim \Gamma\left(a_{1}+\sum_{i=1}^{n} I_{i 1} ; b_{1}+\log \left(1+\frac{t_{n}}{\beta_{1}}\right)\right)
$$

(iii)

$$
\pi\left(\beta_{2} \mid \alpha, \beta_{1}, I, \mathcal{D}_{t}\right) \sim \Gamma\left(a_{3}+n-\sum_{i=1}^{n} I_{i 1} ; b_{3}+\frac{t_{n}^{2}}{2}\right)
$$

(iv)

$$
\begin{gathered}
\pi\left(\beta_{1} \mid \alpha, \beta_{2}, I, \mathcal{D}_{t}\right) \propto \beta_{1}^{a_{2}-1} e^{-b_{2} \beta_{1}} \psi\left(\underline{\beta_{1}}\right) . \\
\text { onde } \quad \psi\left(\underline{\beta_{1}}\right)=\exp \left(-\alpha \log \left(1+\frac{t_{n}}{\beta_{1}}\right)-\sum_{i=1}^{n} I_{i 1} \log \left(\beta_{1}+t_{i}\right)\right) .
\end{gathered}
$$

Observamos que, é preciso o uso do algoritmo Metropolis-Hastings para gerar a variável $\beta_{1}$.

\subsection{Superposição de modelos na presença de uma co- variada.}

\subsubsection{Influência da covariada nas probabilidades de incidência.}

Assumimos agora que temos uma covariável $x$ que poderia estar relacionada com o tipo de input ou com os diferentes programadores. Na presença da covariada $x$, assumimos o modelo de superposição com função intensidade :

$$
\lambda(t \mid \underline{\beta}, \underline{\gamma}, x)=\sum_{j=1}^{J} \theta_{j} \lambda_{j}\left(t \mid \underline{\beta_{j}}\right)
$$


onde, $\underline{\beta}=\left(\beta_{1}, \cdots, \beta_{J}\right), \quad \underline{\gamma}=\left(\gamma_{1}, \cdots, \gamma_{K}\right)$ são parâmetros relacionados com as probabilidades de incidência $\theta_{j}$ de modo que $\sum_{j=1}^{J} \theta_{j}=1$

A ligação logística é usada para as probabilidades de incidência $\theta_{j}, \quad j=1, \cdots, J$

Para o caso particular $\mathrm{J}=2$, temos que:

$$
\lambda\left(t_{i}\right)=\theta_{1 i} \lambda_{1}\left(t_{i}\right)+\theta_{2 i} \lambda_{2}\left(t_{i}\right)
$$

onde,

$$
\begin{aligned}
\theta_{1 i} & =\frac{e^{\gamma+\tau x_{i}}}{1+e^{\gamma+\tau x_{i}}} \\
\theta_{2 i} & =1-\theta_{1 i} .
\end{aligned}
$$

Observamos que neste caso, $\underline{\gamma}=(\gamma, \tau)$

É interessante observar que Yamada and Osaki, 1984 e 1985, consideram a superposição de dois modelos Goel-Okumoto, 1979, cada um deles com função intensidade : $\lambda_{j}(t)=\xi \theta_{j} \beta_{j} e^{-\beta_{j} t}, \quad j=1,2$, onde $\xi>0, \beta_{j}>0,0<\theta_{j}<1$ e $\theta_{1}+\theta_{2}=1$, mas não incluindo covariadas.

\section{Modelo 2: Superposição dos processos Musa e Okumoto com Exponencial} incluindo uma covariada.

Novamente consideramos os processos Musa e Okumoto com Exponencial, mas neste caso incluindo uma covariada $x$, ou seja, usamos uma função intensidade dada por,

$$
\lambda\left(t_{i}\right)=\frac{\alpha}{\beta_{1}+t_{i}} \theta_{1 i}+\beta_{2} t_{i} \theta_{2 i} \quad i=1, \cdots, n .
$$

Assumimos o modelo de falhas truncado. Denotamos o vetor de parâmetros deste modelo por $\underline{\beta_{2}}=\left(\alpha, \beta_{1}, \beta_{2}, \gamma, \tau\right)$. A função de verossimilhança para $\underline{\beta_{2}}$ é, de (2.3), dada por,

$$
L\left(\underline{\beta_{2}} \mid \mathcal{D}_{t}\right)=\left(\prod_{i=1}^{n}\left(\left(\frac{\alpha}{\beta_{1}+t_{i}}\right) \theta_{1 i}+\beta_{2} t_{i} \theta_{2 i}\right)\right) \exp \left(-\theta_{1 n} \alpha \log \left(1+\frac{t_{n}}{\beta_{1}}\right)-\frac{\theta_{2 n} \beta_{2} t_{n}^{2}}{2}\right),
$$


onde,

$$
\begin{aligned}
\theta_{1 n} & =\frac{e^{\gamma+\tau x_{n}}}{1+e^{\gamma+\tau x_{n}}} \\
\mathrm{e} \quad \theta_{2 n} & =1-\theta_{1 n} .
\end{aligned}
$$

Assumimos as seguintes distribuições a priori para os elementos de $\underline{\beta_{2}}$

$$
\begin{aligned}
\alpha & \sim \Gamma\left(a_{1}, b_{1}\right) \\
\beta_{1} & \sim \Gamma\left(a_{2}, b_{2}\right) \\
\beta_{2} & \sim \Gamma\left(a_{3}, b_{3}\right) \\
\gamma & \sim N\left(c_{1}, d_{1}\right) \\
\tau & \sim N\left(c_{2}, d_{2}\right),
\end{aligned}
$$

onde, $a_{1}, a_{2}, a_{3}, b_{1}, b_{2}, b_{3}, c_{1}, c_{2}, d_{1}, d_{2}$ são conhecidas e $N\left(\mu, \sigma^{2}\right)$ denota uma distribuição normal com média $\mu$ e variância $\sigma^{2}$. Assumimos também independência a priori entre os parâmetros.

Introduzimos as variáveis latentes $I_{i j}$, como na seção 2.2. Assim, $I_{i 1} \sim \operatorname{Bernoulli}\left(p_{i 1}\right)$, tal que,

$$
p_{i 1}=\frac{\alpha \theta_{1 i}}{\alpha \theta_{1 i}+\beta_{2} \theta_{2 i} t_{i}\left(\beta_{1}+t_{i}\right)} .
$$

Usando (2.12) e as duas últimas equações de (2.18), obtemos a distribuição a posteriori para $\underline{\beta_{2}}$,

$$
\begin{aligned}
\pi\left(\underline{\beta_{2}} \mid I, \mathcal{D}_{t}\right) \propto & \frac{\left(\prod_{i: I_{i 1}=1}^{n} \theta_{1 i}\right)\left(\prod_{i: I_{i 2}=1}^{n} t_{i} \theta_{2 i}\right)}{\prod_{i: I_{i 1}=1}^{n}\left(\beta_{1}+t_{i}\right)} \\
& \alpha^{a_{1}-1+\sum_{i=1}^{n} I_{i 1}} \beta_{1}^{a_{2}-1} \beta_{2}^{a_{3}-1+n-\sum_{i=1}^{n} I_{i 1}} e^{-b_{2} \beta_{1}} \\
& \exp \left(-\left(b_{1}+\theta_{1 n} \log \left(1+\frac{t_{n}}{\beta_{1}}\right)\right) \alpha-\left(b_{3}+\theta_{2 n} \frac{t_{n}^{2}}{2}\right) \beta_{2}\right) \\
& \exp \left(-\frac{\left(\gamma-c_{1}\right)^{2}}{2 d_{1}}-\frac{\left(\tau-c_{2}\right)^{2}}{2 d_{2}}\right),
\end{aligned}
$$


onde

$$
\begin{aligned}
\theta_{1 i} & =\frac{e^{\gamma+\tau x_{i}}}{1+e^{\gamma+\tau x_{i}}} \\
\theta_{2 i} & =1-\theta_{1 i} .
\end{aligned}
$$

As distribuições condicionais para o algoritmo de Gibbs são dadas por

(i) Construir I dados $\alpha, \beta_{1}, \beta_{2}, \gamma, \tau$ e $\mathcal{D}_{t}$ gerando variáveis aleatórias independentes $I_{i 1}$ a partir de uma distribuição Bernoulli com parâmetros $p_{i 1}$ dados por (2.19).

(ii)

$$
\pi\left(\alpha \mid \beta_{1}, \beta_{2}, \gamma, \tau, I, \mathcal{D}_{t}\right) \sim \Gamma\left(a_{1}+\sum_{i=1}^{n} I_{i 1} ; b_{1}+\theta_{1 n} \log \left(1+\frac{t_{n}}{\beta_{1}}\right)\right)
$$

(iii)

$$
\pi\left(\beta_{2} \mid \alpha, \beta_{1}, \gamma, \tau, I, \mathcal{D}_{t}\right) \sim \Gamma\left(a_{3}+n-\sum_{i=1}^{n} I_{i 1} ; b_{3}+\frac{\theta_{2 n} t_{n}^{2}}{2}\right)
$$

(iv)

$$
\pi\left(\beta_{1} \mid \alpha, \beta_{2}, \gamma, \tau, I, \mathcal{D}_{t}\right) \propto \beta_{1}^{a_{2}-1} e^{-b_{2} \beta_{1}} \psi_{1}\left(\underline{\beta_{2}}\right)
$$

onde,

$$
\psi_{1}\left(\underline{\beta_{2}}\right)=\exp \left(-\alpha \theta_{1 n} \log \left(1+\frac{t_{n}}{\beta_{1}}\right)-\sum_{i=1}^{n} I_{i 1} \log \left(\beta_{1}+t_{i}\right)\right)
$$

(v)

$$
\pi\left(\gamma, \tau \mid \alpha, \beta_{1}, \beta_{2}, I, \mathcal{D}_{t}\right) \propto \exp \left(-\frac{\left(\gamma-c_{1}\right)^{2}}{2 d_{1}}-\frac{\left(\tau-c_{2}\right)^{2}}{2 d_{2}}\right) \psi_{2}\left(\underline{\beta_{2}}\right)
$$

onde,

$$
\psi_{2}\left(\underline{\beta_{2}}\right)=\exp \left(\sum_{i=1}^{n} I_{i 1} \log \theta_{1 i}+\sum_{i=1}^{n} I_{i 2} \log \theta_{2 i}-\theta_{1 n} \alpha \log \left(1+\frac{t_{n}}{\beta_{1}}\right)-\frac{\theta_{2} \beta_{2} t_{n}^{2}}{2}\right) .
$$

Observamos que é necessário o uso do algoritmo de Metropolis-Hastings para gerar as variáveis $\beta_{1}, \gamma$ e $\tau$ 


\subsubsection{Influência da covariada nas probabilidades de incidência e na função intensidade.}

Nesta seção estudamos o caso em que a covariada $x$ influencia tanto as probabilidades de incidência quanto a função intensidade. Assim, consideramos a superposição de processo PPNH com função intensidade dada por,

$$
\lambda(t \mid \underline{\beta}, \underline{\gamma}, \underline{\xi}, x)=\sum_{j=1}^{J} \theta_{j} \lambda_{j}\left(t \mid \underline{\beta_{j}}\right) e^{-\xi_{j} x},
$$

onde, $\underline{\xi}=\left(\xi_{1}, \cdots, \xi_{J}\right)$.

Modelo 3: Superposição dos processos Musa e Okumoto e Exponencial incluindo uma covariada que influencia as probabilidades de incidência e as funções intensidades.

Considerando novamente a superposição dos processos Musa e Okumoto com Exponencial e o modelo de falhas truncado.

Denotamos o vetor de parâmetros por $\underline{\beta_{3}}=\left(\alpha, \beta_{1}, \beta_{2}, \gamma, \tau, \xi_{1}, \xi_{2}\right)$. A função de verossimilhança para $\underline{\beta_{3}}$ é, de (2.3), dada por,

$$
\begin{array}{r}
L\left(\underline{\beta_{3}} \mid \mathcal{D}_{t}\right)=\left(\prod_{i=1}^{n}\left(\left(\frac{\alpha}{\beta_{1}+t_{i}}\right) \theta_{1 i} e^{-\xi_{1} x_{i}}+\beta_{2} t_{i} \theta_{2 i} e^{-\xi_{2} x_{i}}\right)\right) . \\
\cdot \exp \left(-\theta_{1 n} \alpha \log \left(1+\frac{t_{n}}{\beta_{1}}\right) e^{-\xi_{1} x_{i}}-\frac{\theta_{2 n} \beta_{2} t_{n}^{2}}{2} e^{-\xi_{2} x_{i}}\right) .
\end{array}
$$

Usamos as distribuições a priori para $\alpha, \beta_{1}, \beta_{2}, \gamma$ e $\tau$ da forma (2.18) e consideramos as distribuições a priori para $\xi_{1}$ e $\xi_{2}$ dadas por,

$$
\begin{aligned}
& \xi_{1} \sim N\left(c_{3}, d_{3}\right), \\
& \xi_{2} \sim N\left(c_{4}, d_{4}\right) .
\end{aligned}
$$


Novamente introduzimos variáveis latentes $I_{i j}$, definidas como antes, mas com distribuição Bernoulli $\left(p_{i j}\right)$ onde,

$$
p_{i j}=\frac{\theta_{j i} \lambda_{j}\left(t_{i}\right) e^{-\xi_{j} x_{i}}}{\sum_{j=1}^{J} \theta_{i j} \lambda_{j}\left(t_{i}\right) e^{-\xi_{j} x_{i}}}
$$

onde, $j=1, \cdots, J, \quad i=1, \cdots, n$.

No caso particular que estamos considerando bastará usar as variáveis $I_{i 1} \sim \operatorname{Bernoulli}\left(p_{i 1}\right)$ onde,

$$
p_{i 1}=\frac{\theta_{1 i}\left(\frac{\alpha}{\beta_{1}+t_{i}}\right) e^{-\xi_{1} x_{i}}}{\theta_{1 i}\left(\frac{\alpha}{\beta_{1}+t_{i}}\right) e^{-\xi_{1} x_{i}}+\left(1-\theta_{1 i}\right) \beta_{2} t_{i} e^{-\xi_{2} x_{i}}}
$$

para $i=1, \cdots, n$.

A distribuição a posteriori para $\underline{\beta_{3}}$ é dada por,

$$
\begin{aligned}
& \pi\left(\underline{\beta_{3}} \mid I, \mathcal{D}_{t}\right) \propto \frac{\left(\prod_{i: I_{i 1}=1}^{n} \theta_{1 i} e^{-\xi_{1} x_{i}}\right)\left(\prod_{i: I_{i 2}=1}^{n} t_{i} \theta_{2 i} e^{-\xi_{2} x_{i}}\right)}{\prod_{i: I_{i 1}=1}^{n}\left(\beta_{1}+t_{i}\right)} \\
& \alpha^{a_{1}-1+\sum_{i=1}^{n} I_{i 1}} \beta_{1}^{a_{2}-1} \beta_{2}^{a_{3}-1+n-\sum_{i=1}^{n} I_{i 1}} e^{-b_{2} \beta_{1}} \\
& \exp \left(-\frac{\left(\xi_{1}-e_{1}\right)^{2}}{2 f_{1}}-\frac{\left(\xi_{2}-e_{2}\right)^{2}}{2 f_{2}}\right) \\
& \exp \left(-\left(b_{1}+\theta_{1 n} e^{-\xi_{1} x_{n}} \log \left(1+\frac{t_{n}}{\beta_{1}}\right)\right) \alpha-\left(b_{3}+\theta_{2 n} e^{-\xi_{2} x_{n}} \frac{t_{n}^{2}}{2}\right) \beta_{2}\right) \\
& \exp \left(-\frac{\left(\gamma-c_{1}\right)^{2}}{2 d_{1}}-\frac{\left(\tau-c_{2}\right)^{2}}{2 d_{2}}\right) \text {. }
\end{aligned}
$$

As distribuições condicionais para o algoritmo de amostrador de Gibbs são dadas por,

(i) Construir $I_{i 1}$ dados $\underline{\beta_{3}}$ e $\mathcal{D}$ gerando variáveis independentes $\operatorname{Bernoulli}\left(p_{i 1}\right)$, onde $p_{i 1}$ é dado por (2.24).

(ii)

$$
\pi\left(\alpha \mid \beta_{1}, \beta_{2}, \gamma, \tau, \xi_{1}, \xi_{2}, I, \mathcal{D}_{t}\right) \sim \Gamma\left(a_{1}+\sum_{i=1}^{n} I_{i 1} ; b_{1}+\theta_{1 n} e^{-\xi_{1} x_{n}} \log \left(1+\frac{t_{n}}{\beta_{1}}\right)\right)
$$


(iii)

$$
\pi\left(\beta_{2} \mid \alpha, \beta_{1}, \gamma, \tau, \xi_{1}, \xi_{2}, I, \mathcal{D}_{t}\right) \sim \Gamma\left(a_{3}+n-\sum_{i=1}^{n} I_{i 1} ; b_{3}+\frac{\theta_{2 n} e^{-\xi_{2} x_{n}} t_{n}^{2}}{2}\right)
$$

(iv)

$$
\pi\left(\beta_{1} \mid \alpha, \beta_{2}, \gamma, \tau, \xi_{1}, \xi_{2}, I, \mathcal{D}_{t}\right) \propto \beta_{1}^{a_{2}-1} e^{-b_{2} \beta_{1}} \psi_{1}\left(\underline{\beta_{3}}\right),
$$

onde

$$
\psi_{1}\left(\underline{\beta_{3}}\right)=\exp \left(-\alpha \theta_{1 n} e^{-\xi_{1} x_{n}} \log \left(1+\frac{t_{n}}{\beta_{1}}\right)-\sum_{i=1}^{n} I_{i 1} \log \left(\beta_{1}+t_{i}\right)\right) .
$$

(v)

$$
\pi\left(\gamma, \tau \mid \alpha, \beta_{1}, \beta_{2}, \xi_{1}, \xi_{2}, I, \mathcal{D}_{t}\right) \propto \exp \left(-\frac{\left(\gamma-c_{1}\right)^{2}}{2 d_{1}}-\frac{\left(\tau-c_{2}\right)^{2}}{2 d_{2}}\right) \psi_{2}\left(\underline{\beta_{3}}\right),
$$

onde

$$
\begin{aligned}
\psi_{2}\left(\underline{\beta_{3}}\right)= & \exp \left(\sum_{i=1}^{n} I_{i 1} \log \theta_{1 i}-\xi_{1} \sum_{i=1}^{n} x_{i} I_{i 1}+\right. \\
& \sum_{i=1}^{n}\left(1-I_{i 1}\right) \log \theta_{2 i}-\xi_{2} \sum_{i=1}^{n} x_{i}\left(1-I_{i 1}\right)- \\
& \left.\theta_{1 n} e^{-\xi_{1} x_{n}} \alpha \log \left(1+\frac{t_{n}}{\beta_{1}}\right)-\frac{\theta_{2 n} e^{-\xi_{2} x_{n}} \beta_{2} t_{n}^{2}}{2}\right) .
\end{aligned}
$$

(vi)

$$
\pi\left(\xi_{1}, \xi_{2} \mid \alpha, \beta_{1}, \beta_{2}, \gamma, \tau, I, \mathcal{D}_{t}\right) \propto \exp \left(-\frac{\left(\xi_{1}-e_{1}\right)^{2}}{2 f_{1}}-\frac{\left(\xi_{2}-e_{2}\right)^{2}}{2 f_{2}}\right) \psi_{3}\left(\underline{\beta_{3}}\right),
$$

onde

$$
\begin{aligned}
\psi_{3}\left(\underline{\beta_{3}}\right)= & \exp \left(-\xi_{1} \sum_{i=1}^{n} I_{i 1} x_{i}-\xi_{2} \sum_{i=1}^{n} I_{i 2} x_{i}\right. \\
& \left.-\alpha \theta_{1 n} e^{-\xi_{1} x_{n}} \log \left(1+\frac{t_{n}}{\beta_{1}}\right)-\frac{\beta_{2} \theta_{2 n} e^{-\xi_{2} x_{n}} t_{n}^{2}}{2}\right) .
\end{aligned}
$$




\subsection{Algumas considerações relativas às estimativas de Bayes e à seleção de modelos}

O objetivo desta seção é obtermos inferências da função de valor médio

$$
m(t)=\sum_{j=1}^{J} m_{j}(t)
$$

em algum tempo específico $t$.

Um estimador de Bayes para $m(t)$ considerando a função de perda quadrática é dado pela média a posteriori $E\left(m(t) \mid \mathcal{D}_{t}\right)$, a qual podemos aproximar pela estimativa de Monte Carlo usando as $S$ amostras de Gibbs geradas.

As estimativas de Bayes para $m(t)$ para os três modelos vistos nas seções precedentes, são as seguintes,

(i) Modelo 1 .

$$
\widehat{E}\left(m(t) \mid \mathcal{D}_{t}\right)=\frac{1}{S} \sum_{s=1}^{S}\left(\alpha^{(s)} \log \left(1+\frac{t}{\beta_{1}^{(s)}}\right)+\frac{\beta_{2}^{(s)} t^{2}}{2}\right) .
$$

(ii) Modelo 2.

$$
\hat{E}\left(m(t) \mid \mathcal{D}_{t}\right)=\frac{1}{S} \sum_{s=1}^{S}\left(\theta_{i 1}^{(s)} \alpha^{(s)} \log \left(1+\frac{t}{\beta_{1}^{(s)}}\right)+\frac{\theta_{i 2}^{(s)} \beta_{2}^{(s)} t^{2}}{2}\right) .
$$

(iii) Modelo 3.

$$
\widehat{E}\left(m(t) \mid \mathcal{D}_{t}\right)=\frac{1}{S} \sum_{s=1}^{S}\left(\theta_{i 1}^{(s)} \alpha^{(s)} \log \left(1+\frac{t}{\beta_{1}^{(s)}}\right) e^{-\xi_{1}^{(s)} x_{i}}+\frac{\theta_{i 2}^{(s)} \beta_{2}^{(s)} t^{2}}{2} e^{-\xi_{2}^{(s)} x_{i}}\right)
$$

Nesta notação, $\alpha^{(s)}, \beta_{1}^{(s)}, \beta_{2}^{(s)}, \gamma^{(s)}, \tau^{(s)}, \xi_{1}^{(s)}$ e $\xi_{2}^{(s)}$ denotam os valores amostrais para a s-ésima amostra de Gibbs para as variáveis $\alpha, \beta_{1}, \beta_{2}, \gamma, \tau, \xi_{1}$ e $\xi_{2}$ escolhidas da s-ésima amostra de Gibbs. Note-se que, nesta notação também podemos escrever,

$$
\begin{aligned}
\theta_{1 i}^{(s)} & =\frac{e^{\gamma^{(s)}+\tau^{(s)} x_{i}}}{1+e^{\gamma^{(s)}+\tau^{(s)} x_{i}}} \\
\theta_{2 i}^{(s)} & =1-\theta_{1 i}^{(s)} .
\end{aligned}
$$


A função de sobrevivência preditiva avaliada a uma distância $t$ de $t_{n}$ para a superposição de processos PPNH é dada por (veja, Kuo e Yang, 1996).

$$
E\left(S(t) \mid \mathcal{D}_{t}\right)=\int \exp \left(-m\left(t_{n}+t\right)+m\left(t_{n}\right)\right) \pi\left(\underline{\beta} \mid \mathcal{D}_{t}\right) d \underline{\beta} .
$$

As estimativas de Monte Carlo para os três modelos que estamos analisando, são as seguintes,

(i) Modelo 1.

$$
\begin{aligned}
\hat{E}\left(S(t) \mid \mathcal{D}_{t}\right)= & \frac{1}{S} \sum_{s=1}^{S} \exp \left(-\alpha^{(s)} \log \left(1+\frac{t_{n}+t}{\beta_{1}^{(s)}}\right)-\frac{\beta_{2}^{(s)}\left(t_{n}+t\right)^{2}}{2}+\right. \\
& \left.\alpha^{(s)} \log \left(1+\frac{t_{n}}{\beta_{1}^{(s)}}\right)+\frac{\beta_{2}^{(s)} t^{2}}{2}\right) .
\end{aligned}
$$

(ii) Modelo 2.

$$
\begin{aligned}
\widehat{E}\left(S(t) \mid \mathcal{D}_{t}\right)= & \frac{1}{S} \sum_{s=1}^{S} \exp \left(-\theta_{1 n}^{(s)} \alpha^{(s)} \log \left(1+\frac{t_{n}+t}{\beta_{1}^{(s)}}\right)-\frac{\theta_{2 n}^{(s)} \beta_{2}^{(s)}\left(t_{n}+t\right)^{2}}{2}+\right. \\
& \left.\theta_{1 n}^{(s)} \alpha^{(s)} \log \left(1+\frac{t_{n}}{\beta_{1}^{(s)}}\right)+\frac{\theta_{2 n}^{(s)} \beta_{2}^{(s)} t^{2}}{2}\right) .
\end{aligned}
$$

(iii) Modelo 3 .

$$
\begin{aligned}
\hat{E}\left(S(t) \mid \mathcal{D}_{t}\right)= & \frac{1}{S} \sum_{s=1}^{S} \exp \left(-\theta_{1 n}^{(s)} \alpha^{(s)} \log \left(1+\frac{t_{n}+t}{\beta_{1}^{(s)}}\right) e^{-\xi_{1}^{(s)} x_{i}}-\right. \\
& \frac{\theta_{2 n}^{(s)} \beta_{2}^{(s)}\left(t_{n}+t\right)^{2}}{2} e^{-\xi_{2}^{(s)} x_{i}}+ \\
& \left.\theta_{1 n}^{(s)} \alpha^{(s)} \log \left(1+\frac{t_{n}}{\beta_{1}^{(s)}}\right) e^{-\xi_{1}^{(s)} x_{i}}+\frac{\theta_{2 n}^{(s)} \beta_{2}^{(s)} t^{2}}{2} e^{-\xi_{2}^{(s)} x_{i}}\right) .
\end{aligned}
$$

Para a seleção de modelos, podemos considerar (veja, Raftery, 1996) a função de verossimilhança marginal do conjunto de dados $\mathcal{D}_{t}$ para um modelo $\mathrm{M}$, escolhendo o 
modelo tal como vai ser indicado no capítulo 5. Um exemplo numérico è desenvolvido no capítulo 6. 


\section{Capítulo 3}

\section{Uso da distribuição Weibull-}

\section{exponenciada para modelar funções intensidade na forma de banheira}

\subsection{Introdução}

Quando observamos que existe um número desconhecido de falhas, $N$, no começo do processo de teste do software e modelamos os tempos de falha como as $n$ primeiras estatísticas de ordem provenientes de $N$ observações i.i.d. com f.d.p., $f$, e F.D.A., $F$ (um modelo GOS), podemos considerar a função de valor médio dada por:

$$
m(t)=\xi F(t)
$$

onde $N$ é distribuída como uma variável de Poisson com média $\xi$. Observamos que neste caso, $\lim _{t \rightarrow \infty} m(t)$ é finito e este processo é do tipo PPNH-I.

De (3.1), observamos que se $F(t)=1-e^{-\beta t}$, temos o processo de Goel e Okumoto, (veja, Goel e Okumoto, 1979), ou seja, o modelo GOS exponencial. Se $F(t)=1-e^{-\beta t^{\alpha}}$, temos o processo de Goel, 1983, ou seja um processo GOS Weibull. No caso, em que 
$F(t)=1-(1+\beta t) e^{-\beta t}$, temos o processo Ohba-Yamada (veja, Yamada e Osaki, 1984). Se $F(t)=I_{k}\left(\beta t^{\alpha}\right)$, onde $I_{k}(s)$ é a integral gama incompleta, dada por:

$$
I_{k}(s)=\frac{1}{\Gamma(k)} \int_{0}^{s} x^{k-1} e^{-x} d x,
$$

temos um modelo GOS gama generalizado (veja, Achcar, Dey e Niverthy, 1998).

Nas situações onde são introduzidas novas falhas durante o período de debugging, precisamos usar os modelos do tipo RVS, onde

$$
m(t)=-\ln (1-F(t))
$$

e tal que $\quad m(t) \rightarrow \infty$ quando $t \rightarrow \infty$.

Como casos particulares deste tipo de processos, temos o processo de Musa e Okumoto, (veja, Musa, 1984), onde $\lambda(t)=\alpha /(t+\beta)$, ou o processo de Duane, (veja, Duane, 1966) onde $\lambda(t)=\alpha \beta t^{\alpha-1}$ e o processo de Cox e Lewis (veja, Cox e Lewis, 1966) onde, $\lambda(t)=\exp (\alpha+\beta t)$.

Quando a função intensidade é assumida uma função não-monótona, podemos considerar a superposição de vários processos independentes PPNH, onde cada um deles tem função intensidade simples (veja, por exemplo, Kuo e Yang, 1996).

Neste capítulo consideramos o uso de métodos MCMC para obter inferências Bayesianas para o caso particular de processos do tipo PPNH-II : O uso da distribuição Weibullexponenciada (veja, Mudholkar et al., 1982 ou Cancho, V.G., Bolfarine, H., e Achcar, J.A., 1999) para a função intensidade $\lambda(t)$. Com esta escolha para $\lambda(t)$, podemos ter funções intensidades constantes, monótonas e não-monótonas. Também introduzimos uma alternativa para a escolha de função intensidade Weibull-exponenciada, ao considerarmos a superposição de processos PPNH . 


\subsection{Um processo do tipo PPNH-II assumindo funções intensidades Weibull-exponenciada}

A função intensidade, $\lambda(t)$, para a distribuição Weibull-exponenciada é dada por:

$$
\lambda(t)=\frac{\alpha \theta\left[1-\exp \left(-(t / \sigma)^{\alpha}\right)\right]^{\theta-1} \exp \left(-(t / \sigma)^{\alpha}\right)(t / \sigma)^{\alpha-1}}{\sigma\left(1-\left[1-\exp \left(-(t / \sigma)^{\alpha}\right)\right]^{\theta}\right)} .
$$

A grande flexibilidade deste modelo para ajustar dados de confiabilidade de software é dada pelas diferentes formas que a função intensidade pode tomar, isto é:

(i) Se $\alpha \geq 1 \quad$ e $\quad \alpha \theta \geq 1$, temos uma função intensidade monótona crescente;

(ii) Se $\alpha \leq 1 \quad e \quad \alpha \theta \leq 1$, temos uma função intensidade monótona decrescente;

(iii) Se $\alpha>1 \quad e \quad \alpha \theta<1$, temos uma função intensidade com forma de banheira. Observar que neste caso $0<\theta<1$;

(iv) Se $\alpha<1 \quad e \quad \alpha \sigma>1$, temos uma função intensidade unimodal.

A função de valor médio , $m(t)$, é dada por (3.2), onde:

$$
F(t)=\left[1-\exp \left(-(t / \sigma)^{\alpha}\right)\right]^{\theta}
$$

Assumimos o modelo de falhas truncado, onde os tempos de falha ordenados são denotados por $t_{1}, t_{2}, \cdots, t_{n}$.

A função de verossimilhança é dada por:

$$
L(\alpha, \theta, \sigma \mid \mathcal{D})=\left(\prod_{1}^{n} \lambda\left(t_{i}\right)\right) \exp \left(-m\left(t_{n}\right)\right)
$$

onde $\mathcal{D}$ é o conjunto de dados. 
Substituindo a função intensidade e a função de valor médio por suas respectivas expressões, temos:

$$
L(\alpha, \theta, \sigma \mid \mathcal{D})=\frac{\alpha^{n} \theta^{n} A(\alpha, \theta, \sigma) S\left(t_{n}\right)}{\sigma^{n \alpha} \prod_{1}^{n} S\left(t_{i}\right)},
$$

onde $\left.S\left(t_{i}\right)=1-F\left(t_{i}\right)=1-\left(1-\exp \left(-\left(t_{i} / \sigma\right)^{\alpha}\right)\right)\right)^{\theta}$,

e $\quad A(\alpha, \theta, \sigma)=\prod_{1}^{n} t_{i}^{\alpha-1} \exp \left(-\left(t_{i} / \sigma\right)^{\alpha}\right)\left(1-\exp \left(-\left(t_{i} / \sigma\right)^{\alpha}\right)\right)^{\theta-1}$.

Para fazer inferência Bayesiana, assumimos as seguintes densidades a priori para $\alpha, \theta$ e $\sigma:$

$$
\begin{aligned}
\alpha & \sim \Gamma\left(a_{1}, b_{1}\right), \\
\theta & \sim \Gamma\left(a_{2}, b_{2}\right), \\
\sigma & \sim \Gamma\left(a_{3}, b_{3}\right),
\end{aligned}
$$

onde $a_{1}, a_{2}, a_{3}, b_{1}, b_{2}$ e $b_{3}$ são constantes conhecidas, e $\Gamma(a, b)$ denota a distribuição gama com média $a / b$ e variância $a / b^{2}$. Também assumimos independência entre os parâmetros $\alpha, \theta$ e $\sigma$.

A densidade conjunta a posteriori para $\alpha, \theta$ e $\sigma$ é dada por:

$$
\begin{aligned}
\pi(\alpha, \theta, \sigma \mid \mathcal{D}) \propto & \alpha^{a_{1}+n-1} \theta^{a_{2}+n-1} \sigma^{a_{3}-n \alpha-1} \\
& \exp \left(-b_{1} \alpha-b_{2} \theta-b_{3} \sigma\right) \frac{A(\alpha, \theta, \sigma) b(\alpha, \theta, \sigma)}{B(\alpha, \theta, \sigma)},
\end{aligned}
$$

onde $\quad b(\alpha, \theta, \sigma)=S\left(t_{n}\right), \quad B(\alpha, \theta, \sigma)=\prod_{1}^{n} S\left(t_{i}\right)$.

As densidades condicionais a posteriori para o algoritmo de Gibbs são dadas por:

(i)

$$
\pi(\alpha \mid \theta, \sigma, \mathcal{D}) \propto \alpha^{a_{1}-1} e^{-b_{1} \alpha} \psi_{1}(\alpha, \theta, \sigma),
$$

onde

$$
\psi_{1}(\alpha, \theta, \sigma)=\exp (n \ln \alpha+\ln A(\alpha, \theta, \sigma)+\ln b(\alpha, \theta, \sigma)-\ln B(\alpha, \theta, \sigma)) .
$$


(ii)

$$
\pi(\theta \mid \alpha, \sigma, \mathcal{D}) \propto \theta^{a_{2}-1} e^{-b_{2} \theta} \psi_{2}(\alpha, \theta, \sigma)
$$

onde

$$
\psi_{2}(\alpha, \theta, \sigma)=\exp (n \ln \theta+\ln A(\alpha, \theta, \sigma)+\ln b(\alpha, \theta, \sigma)-\ln B(\alpha, \theta, \sigma)) .
$$

(iii)

$$
\pi(\sigma \mid \alpha, \theta, \mathcal{D}) \propto \sigma^{a_{3}-1} e^{-b_{3} \sigma} \psi_{3}(\alpha, \theta, \sigma)
$$

onde

$$
\psi_{3}(\alpha, \theta, \sigma)=\exp (-n \alpha \ln \sigma+\ln A(\alpha, \theta, \sigma)+\ln b(\alpha, \theta, \sigma)-\ln B(\alpha, \theta, \sigma)) .
$$

Observar que para gerar as três variáveis $\alpha, \theta$ e $\sigma$ é necessário usar o algoritmo de Metropolis-Hastings.

\subsection{Superposição de processos PPNH considerando a introdução de probabilidade de incidência}

Assumimos agora, que o modelo de superposição de processos PPNH com função intensidade ,

$$
\lambda(t \mid \underline{\theta}, \underline{\beta})=\sum_{j=1}^{J} \theta_{j} \lambda_{j}\left(t \mid \underline{\beta_{j}}\right),
$$

onde as probabilidades de incidência $\theta_{j}$ satisfazem,

$$
\sum_{j=1}^{J} \theta_{j}=1 \quad \text { e } \quad \underline{\theta}=\left(\theta_{1}, \theta_{2}, \cdots, \theta_{J}\right) .
$$


Um caso particular é a generalização da função intensidade de Musa e Okumoto com exponencial dada por:

$$
\lambda(t \mid \theta, \underline{\beta})=\frac{\theta \alpha}{\beta_{1}+t}+(1-\theta) \beta_{2} t
$$

onde $\theta_{1}=\theta \quad$ quando $\quad J=2$.

Neste caso, a função de valor médio, $m(t)$ é dada por:

$$
m(t \mid \underline{\theta}, \underline{\beta})=\theta \alpha \ln \left(1+\frac{t}{\beta_{1}}\right)+(1-\theta) \beta_{2} \frac{t^{2}}{2} .
$$

Assumindo o modelo de falhas truncado, a função de verossimilhança para $\theta, \alpha, \beta_{1}$ e $\beta_{2}$ é dada por,

$$
\begin{aligned}
L\left(\theta, \alpha, \beta_{1}, \beta_{2} \mid \mathcal{D}\right)= & \left(\prod_{i=1}^{n}\left(\frac{\alpha \theta}{\beta_{1}+t_{i}}+(1-\theta) \beta_{2} t_{i}\right)\right) \\
& \exp \left(-\theta \alpha \ln \left(1+\frac{t_{n}}{\beta_{1}}\right)-(1-\theta) \frac{\beta_{2} t_{n}^{2}}{2}\right) .
\end{aligned}
$$

Assumimos as seguintes distribuições a priori para $\alpha, \beta_{1}, \beta_{2}$ e $\theta$

$$
\begin{aligned}
\alpha & \sim \Gamma\left(a_{1}, b_{1}\right) \\
\beta_{1} & \sim \Gamma\left(a_{2}, b_{2}\right) \\
\beta_{2} & \sim \Gamma\left(a_{3}, b_{3}\right) \\
\theta & \sim \beta\left(a_{4}, b_{4}\right),
\end{aligned}
$$

onde $a_{i} \quad e \quad b_{i} \quad$ para $\quad i=1,2,3,4$ são constantes conhecidas.

Então a distribuição conjunta a posteriori para $\alpha, \beta_{1}, \beta_{2}$ e $\theta$ é dada por:

$$
\begin{aligned}
\pi\left(\alpha, \beta_{1}, \beta_{2}, \theta \mid \mathcal{D}\right) \propto & \left(\prod_{i=1}^{n}\left(\frac{\alpha \theta}{\beta_{1}+t_{i}}+(1-\theta) \beta_{2} t_{i}\right)\right) \\
& \exp \left(-b_{1} \alpha-b_{2} \beta_{1}-b_{3} \beta_{2}-\theta \alpha \ln \left(1+\frac{t_{n}}{\beta_{1}}\right)-(1-\theta) \frac{\beta_{2} t_{n}^{2}}{2}\right) \\
& \alpha_{1}^{a_{1}-1} \beta_{1}^{a_{2}-1} \beta_{2}^{a_{3}-1} \theta^{a_{4}-1}(1-\theta)^{b_{4}-1} .
\end{aligned}
$$


Consideramos a introdução de variáveis latentes $I_{i j}$ como na seção 2.2. Assim,

$$
p_{i 1}=\frac{\theta \alpha /\left(\beta_{1}+t_{i}\right)}{\theta \alpha /\left(\beta_{1}+t_{i}\right)+(1-\theta) \beta_{2} t_{i}} .
$$

Logo,

$$
\pi\left(\underline{I_{1}}, \cdots, \underline{I_{n}} \mid \underline{\beta}, \underline{\theta}, \mathcal{D}\right) \propto \frac{(\theta \alpha)^{\sum_{i=1}^{n} I_{i 1}}\left((1-\theta) \beta_{2}\right)^{\sum_{i=1}^{n} I_{i 2}}\left(\prod_{i=1}^{n} t_{i}^{I_{i 2}}\right)}{\left(\prod_{i: I_{i 1}=1}^{n}\left(\beta_{1}+t_{i}\right)\right)\left(\prod_{i=1}^{n}\left(\theta \alpha /\left(\beta_{1}+t_{i}\right)+(1-\theta) \beta_{2} t_{i}\right)\right.},
$$

onde $I_{i 1}+I_{i 2}=1$.

A distribuição conjunta a posteriori para $\alpha, \beta_{1}, \beta_{2}$ e $\theta$ dado $I=\left(\underline{I_{1}}, \cdots, \underline{I_{n}}\right)$ e $\mathcal{D}$ é dada por,

$$
\begin{aligned}
\pi\left(\alpha, \beta_{1}, \beta_{2}, \theta \mid I, \mathcal{D}\right) \propto & \frac{1}{\left(\prod_{i: I_{i 1}=1}^{n}\left(\beta_{1}+t_{i}\right)\right)} \alpha^{a_{1}-1+\sum_{i=1}^{n} I_{i 1}} \beta_{1}^{a_{2}-1} \beta_{2}^{a_{3}-1+\sum_{i=1}^{n} I_{i 2}} \\
& \theta^{a_{4}-1+\sum_{i=1}^{n} I_{i 1}}(1-\theta)^{b_{4}-1+\sum_{i=1}^{n} I_{i 2}} e^{-b_{1} \alpha} e^{-b_{2} \beta_{1}} e^{-b_{3} \beta_{2}} \\
& \exp \left(-\theta \alpha \ln \left(1+\frac{t_{n}}{\beta_{1}}\right)-(1-\theta) \frac{\beta_{2} t_{n}^{2}}{2}\right) .
\end{aligned}
$$

As distribuições condicionais para o algoritmo de Gibbs são as seguintes:

(i) Construir $I=\left(\underline{I_{1}}, \cdots, \underline{I_{n}}\right)^{T}$ (uma matriz nx2) dados $\underline{\beta}$ e $\mathcal{D}$ para gerar variáveis aleatórias independentes $I_{i 1}$ com distribuição Bernoulli com parâmetros $p_{i 1}$ dados por (3.11).

(ii)

$$
\pi\left(\alpha \mid \beta_{1}, \beta_{2}, \theta, I, \mathcal{D}\right) \sim \Gamma\left(a_{1}+\sum_{i=1}^{n} I_{i 1} ; b_{1}+\theta \ln \left(1+\frac{t_{n}}{\beta_{1}}\right)\right) .
$$

(iii)

$$
\pi\left(\beta_{1} \mid \alpha, \beta_{2}, \theta, I, \mathcal{D}\right) \propto \beta_{1}^{a_{2}-1} e^{-b_{2} \beta_{1}} \psi_{1}\left(\alpha, \beta_{1}, \beta_{2}, \theta\right),
$$


onde

$$
\psi_{1}\left(\alpha, \beta_{1}, \beta_{2}, \theta\right)=\exp \left(-\theta \alpha \ln \left(1+\frac{t_{n}}{\beta_{1}}\right)-\sum_{i=1}^{n} I_{i 1} \ln \left(\beta_{1}+t_{i}\right)\right)
$$

(iv)

$$
\pi\left(\beta_{2} \mid \alpha, \beta_{1}, \theta, I, \mathcal{D}\right) \sim \Gamma\left(a_{3}+n-\sum_{i=1}^{n} I_{i 1} ; b_{3}+(1-\theta) \frac{t_{n}^{2}}{2}\right) .
$$

(v)

$$
\pi\left(\theta \mid \alpha, \beta_{1}, \beta_{2}, I, \mathcal{D}\right) \propto \theta^{a_{4}-1+\sum_{i=1}^{n} I_{i 1}}(1-\theta)^{b_{4}+n-1-\sum_{i=1}^{n} I_{i 1}} \psi_{2}\left(\alpha, \beta_{1}, \beta_{2}, \theta\right)
$$

onde

$$
\psi_{2}\left(\alpha, \beta_{1}, \beta_{2}, \theta\right)=\exp \left(-\theta \alpha \ln \left(1+\frac{t_{n}}{\beta_{1}}\right)-(1-\theta) \frac{\beta_{2} t_{n}^{2}}{2}\right)
$$

Observamos que para gerar as variáveis $\beta_{1}$ e $\theta$ precisamos do uso do algoritmo Metropolis-Hastings.

\subsection{Inferência Bayesiana e escolha do modelo}

No capítulo anterior, usamos a função de verossimilhança marginal para obter estimativas de Bayes para $m(t)$, usando a função de perda quadrática, mediante o uso de amostras de Gibbs. Do mesmo modo, agora podemos calcular $E(m(t) \mid \mathcal{D})$ e $E(\lambda(t) \mid \mathcal{D})$. 


\section{Capítulo 4}

\section{Superposição de processos PPNH dependentes}

\subsection{Introdução}

Estudamos até agora o problema da superposição de processos PPNH sob a suposição de independência dos processos componentes. Esta é uma hipótese conveniente sob o ponto de vista matemático, porém não é uma suposição que seja muito sustentável no mundo real. Daí, a importância de começarmos a resolver o problema da superposição de processos no caso em que os processos componentes não são necessariamente independentes entre si.

\subsection{Função de verossimilhança}

Nesta seção, faremos uso dos processos de Poisson bivariados. Assim, para esclarecer alguns detalhes recomendamos ver também o Apêndice C e o texto de Kocherlakota,S. e Kocherlakota,K., 1992. Consideramos os processos $M_{1}(t)$ e $M_{2}(t)$ que representam o 
número de erros encontrados num teste no intervalo $[0, t) . \quad \lambda_{1}(t)$ e $\quad \lambda_{2}(t)$ representam as funções intensidades dos processos $M_{1}(t)$ e $M_{2}(t)$, respectivamente. Como já foi indicado nos capítulos anteriores, esta notação inclui em forma subjacente o conjunto de parâmetros $\underline{\beta}_{1}=\left(\beta_{11}, \cdots, \beta_{1 J_{1}}\right) \quad e \quad \underline{\beta}_{2}=\left(\beta_{21}, \cdots, \beta_{2 J_{2}}\right)$. Assim, usamos a notação $\lambda_{1}(t)$ no lugar de $\lambda_{1}\left(t \mid \underline{\beta}_{1}\right)$, mas com o compromisso de lembrarmos que por trás temos o conjunto de parâmetros. Também vale a pena lembrarmos que o processo de superposição

$$
M(t)=M_{1}(t)+M_{2}(t)
$$

conta o número total de erros encontrados no intervalo $[0, t)$.

O processo PPNH bivariado $\left(M_{1}(t), M_{2}(t)\right)$ é tal que $M_{i}(t)$ é um processo PPNH com função de valor médio $m_{i}(t)$, onde $m_{i}(t)=E\left[M_{i}(t)\right]=m_{i}^{*}(t)+m_{3}(t), \quad i=1,2$, e tal que,

$$
\begin{aligned}
& M_{1}(t)=X(t)+U(t) \\
& M_{2}(t)=Y(t)+U(t),
\end{aligned}
$$

onde, $X(t), Y(t)$ e $U(t)$ são processos PPNH independentes com funções de valor médio $m_{1}^{*}(t), m_{2}^{*}(t)$ e $m_{3}(t)$, respectivamente.

Também, podemos notar que:

$$
\operatorname{Cov}\left(M_{1}(t), M_{2}(t)\right)=\operatorname{Var}(U(t))=m_{3}(t) \quad \forall t \geq 0,
$$

e que,

$$
\rho(t)=\rho\left(M_{1}(t), M_{2}(t)\right)=\frac{m_{3}(t)}{\left[m_{1}(t) m_{2}(t)\right]^{1 / 2}} \quad \forall t \geq 0 .
$$

Como $m_{3}(t) \geq 0$, concluímos que $\rho(t)$ é não negativo $\forall t \geq 0$.

A função geradora para probabilidades ( F.G.P. ) de $(X(t), Y(t), U(t))$ é dada por,

$$
\begin{aligned}
\phi\left(t_{1}, t_{2}, t_{3}\right) & =E\left[t_{1}^{X(t)} t_{2}^{Y(t)} t_{3}^{U(t)}\right] \\
& =\exp \left(-\left[m_{1}^{*}(t)+m_{2}^{*}(t)+m_{3}(t)\right]+m_{1}^{*}(t) t_{1}+m_{2}^{*}(t) t_{2}+m_{3}(t) t_{3}\right) .
\end{aligned}
$$


Podemos encontrar a F.G.P. da distribuição conjunta de $\left(M_{1}(t), M_{2}(t)\right)$ fazendo $t_{1}=s_{1}, t_{2}=s_{2}, t_{3}=s_{1} s_{2}$. Assim,

$$
\begin{aligned}
\phi\left(s_{1}, s_{2}\right) & =E\left[s_{1}^{M_{1}(t)} s_{2}^{M_{2}(t)}\right] \\
& =\exp \left(-\left[m_{1}^{*}(t)+m_{2}^{*}(t)+m_{3}(t)\right]+m_{1}^{*}(t) s_{1}+m_{2}^{*}(t) s_{2}+m_{3}(t) s_{1} s_{2}\right) .
\end{aligned}
$$

Logo, para obtermos a F.G.P. de $M(t)$, fazemos $s_{1}=s_{2}=s$

$$
\begin{aligned}
\phi_{M(t)}(s) & =E\left[s^{M(t)}\right] \\
& =\exp \left(-\left[m_{1}^{*}(t)+m_{2}^{*}(t)+m_{3}(t)\right]+\left[m_{1}^{*}(t)+m_{2}^{*}(t)\right] s+m_{3}(t) s^{2}\right) .
\end{aligned}
$$

Lema 4.2.1 A f.d.p. de $M(t)$ pode ser escrita na seguinte forma,

$$
\begin{aligned}
f_{M(t)}(m) & =\left[\frac{e^{-\left(m_{1}^{*}(t)+m_{2}^{*}(t)\right)}\left(m_{1}^{*}(t)+m_{2}^{*}(t)\right)^{m}}{m !}\right] \varphi(m), \quad m=0,1,2, \cdots, \\
\text { onde, } \quad \varphi(m) & =e^{-m_{3}(t)}\left(1+\sum_{j=1}^{\left[\frac{1}{2} m\right]} C_{m, j} m_{3}^{j}(t)\right) \\
e, \quad C_{m, j} & =\frac{\left(m_{1}^{*}(t)+m_{2}^{*}(t)\right)^{-2 j} m !}{(m-2 j) ! j !} .
\end{aligned}
$$

\section{Prova}

Kemp, C.D. e Kemp, A.W., 1965, introduziram a distribuição de Poisson generalizada, que é conhecida com o nome de distribuição de Hermite (veja Apêndice B), e cuja F.G.P. é dada por,

$$
\phi_{H}(s)=\exp \left[a_{1}(s-1)+a_{2}\left(s^{2}-1\right)\right]
$$

Kempt e Kempt provaram que a f.d.p. correspondente é dada por,

$$
\begin{aligned}
f_{H}(h) & =P[H=h] \\
& =\exp \left[a_{1}+a_{2}\right] \sum_{j=0}^{\left[\frac{1}{2} h\right]} \frac{a_{1}^{h-2 j} a_{2}^{j}}{(h-2 j) ! j !} \quad h=0,1,2, \cdots,
\end{aligned}
$$

ou seja, a distribuição de Hermite para $\mathrm{H}$. 
Usando (4.4), temos:

$$
\phi_{M(t)}(s)=\exp \left[\left(m_{1}^{*}(t)+m_{2}^{*}(t)\right)(s-1)+m_{3}(t)\left(s^{2}-1\right)\right]
$$

Assim, $a_{1}=m_{1}^{*}(t)+m_{2}^{*}(t), \quad a_{2}=m_{3}(t)$

Logo, a partir de (4.6) obtemos,

$$
\begin{aligned}
f_{M(t)}(m) & =P[M(t)=m] \\
& =\exp \left[-\left(m_{1}^{*}(t)+m_{2}^{*}(t)+m_{3}(t)\right)\right] \sum_{j=0}^{\left[\frac{1}{2} m\right]} \frac{\left(m_{1}^{*}(t)+m_{2}^{*}(t)\right)^{m-2 j} m_{3}^{j}(t)}{(m-2 j) ! j !}
\end{aligned}
$$

Portanto,

$$
f_{M(t)}(m)=\left[\frac{e^{-\left(m_{1}^{*}(t)+m_{2}^{*}(t)\right)}\left(m_{1}^{*}(t)+m_{2}^{*}(t)\right)^{m}}{m !}\right] e^{-m_{3}(t)} S(j)
$$

onde

$$
S(j)=\sum_{j=0}^{\left[\frac{1}{2} m\right]}\left[\frac{\left(m_{1}^{*}(t)+m_{2}^{*}(t)\right)^{-2 j} m !}{(m-2 j) ! j !}\right] m_{3}^{j}(t)
$$

e, desde que, $S(0)=1$, concluímos a prova do Lema 4.2.1.

Podemos concluir que $M(t) \sim \operatorname{Poisson}\left(m_{1}^{*}(t)+m_{2}^{*}(t)\right)$ se e somente se $m_{3}(t)=0$ e, neste caso, $M_{1}(t)$ e $M_{2}(t)$ são processos independentes e,

$$
m_{1}(t)=m_{1}^{*}(t) \quad \text { e } \quad m_{2}(t)=m_{2}^{*}(t) \quad \forall t \geq 0
$$

\section{Lema $\quad 4.2 .2$}

$$
\operatorname{Cov}\left(M_{1}(t)-M_{1}\left(t_{n}\right), M_{2}(t)-M_{2}\left(t_{n}\right)\right)=m_{3}(t)-m_{3}\left(t_{n}\right) \quad \text { se } \quad 0<t_{n}<t .
$$

\section{Prova}

Como, $0<t_{n}<t$ e já que o processo $U(t)$ tem a propriedade de incrementos independentes, temos que:

$$
\operatorname{Cov}\left(U(t)-U\left(t_{n}\right), U\left(t_{n}\right)\right)=0
$$


Logo,

$$
\operatorname{Cov}\left(U(t), U\left(t_{n}\right)\right)=\operatorname{Var}\left(U\left(t_{n}\right)\right)=m_{3}\left(t_{n}\right) .
$$

Por outro lado,

$$
\begin{aligned}
\operatorname{Cov}\left(M_{1}(t)-M_{1}\left(t_{n}\right), M_{2}(t)-M_{2}\left(t_{n}\right)\right)= & \operatorname{Cov}\left(M_{1}(t), M_{2}(t)\right)-\operatorname{Cov}\left(M_{1}(t), M_{2}\left(t_{n}\right)\right) \\
& -\operatorname{Cov}\left(M_{1}\left(t_{n}\right), M_{2}(t)\right)+\operatorname{Cov}\left(M_{1}\left(t_{n}\right), M_{2}\left(t_{n}\right)\right) \\
= & m_{3}(t)-\operatorname{Cov}\left(M_{1}(t), M_{2}\left(t_{n}\right)\right) \\
& \left.-\operatorname{Cov}\left(M_{1}\left(t_{n}\right), M_{2}(t)\right)+m_{3}\left(t_{n}\right)\right)
\end{aligned}
$$

onde,

$$
\operatorname{Cov}\left(M_{1}(t), M_{2}\left(t_{n}\right)\right)=\operatorname{Cov}(X(t)+U(t), Y(t)+U(t))=\operatorname{Cov}\left(U(t), U\left(t_{n}\right)\right) .
$$

Usando (4.8), temos que:

$$
\operatorname{Cov}\left(M_{1}(t), M_{2}\left(t_{n}\right)\right)=m_{3}\left(t_{n}\right)
$$

Similarmente,

$$
\operatorname{Cov}\left(M_{1}\left(t_{n}\right), M_{2}(t)\right)=m_{3}\left(t_{n}\right) .
$$

Logo, de (4.9), (4.10) e (4.11) concluímos o resultado.

\section{Lema 4.2 .3}

$$
\begin{aligned}
P\left[T_{n+1}>t \mid T_{n}=t_{n}\right]= & \exp \left(-\left(m_{1}(t)+m_{2}(t)-m_{3}(t)\right)\right) \\
& \exp \left(m_{1}\left(t_{n}\right)+m_{2}\left(t_{n}\right)-m_{3}\left(t_{n}\right)\right) .
\end{aligned}
$$

\section{Prova}

$$
P\left[T_{n+1}>t \mid T_{n}=t_{n}\right]=P\left[M(t)-M\left(t_{n}\right)=0\right]
$$


Mas,

$$
\begin{aligned}
M(t)-M\left(t_{n}\right) & =M_{1}(t)+M_{2}(t)-M_{1}\left(t_{n}\right)-M_{2}\left(t_{n}\right) \\
& =\left[M_{1}(t)-M_{1}\left(t_{n}\right)\right]+\left[M_{2}(t)-M_{2}\left(t_{n}\right)\right],
\end{aligned}
$$

onde, $M_{i}(t)-M_{i}\left(t_{n}\right)$ é um processo PPNH com função de valor médio $m_{i}(t)-m_{i}\left(t_{n}\right)$, para $i=1,2$. Assim, $M(t)-M\left(t_{n}\right)$ é uma superposição de dois processos PPNH tal que satisfaz o Lema 4.2.2.

Logo, aplicamos o Lema 4.2 .1 ao processo $M(t)-M\left(t_{n}\right)$ para obter, $P\left[M(t)-M\left(t_{n}\right)=0\right]=\exp \left[-\left(m_{1}(t)+m_{2}(t)-m_{3}(t)-m_{1}\left(t_{n}\right)-m_{2}\left(t_{n}\right)+m_{3}\left(t_{n}\right)\right)\right]$ e assim concluímos o resultado.

Lema 4.2 .4

$$
\begin{aligned}
f\left(t_{i} \mid t_{i-1}\right)= & \left(\lambda_{1}\left(t_{i}\right)+\lambda_{2}\left(t_{i}\right)-\lambda_{3}\left(t_{i}\right)\right) \\
& \exp \left(-\left(m_{1}\left(t_{i}\right)+m_{2}\left(t_{i}\right)-m_{3}\left(t_{i}\right)\right)\right) \\
& \exp \left(m_{1}\left(t_{i-1}\right)+m_{2}\left(t_{i-1}\right)-m_{3}\left(t_{i-1}\right)\right) .
\end{aligned}
$$

\section{Prova}

$$
\begin{aligned}
f\left(t_{i} \mid t_{i-1}\right)= & -\frac{d P\left[T_{i}>t_{i} \mid T_{i-1}=t_{i-1}\right]}{d t_{i}} \\
= & -\frac{d}{d t_{i}}\left(\exp \left(-\left(m_{1}\left(t_{i}\right)+m_{2}\left(t_{i}\right)-m_{3}\left(t_{i}\right)\right)\right) \exp \left(m_{1}\left(t_{i-1}\right)+m_{2}\left(t_{i-1}\right)-m_{3}\left(t_{i-1}\right)\right)\right) \\
= & \exp \left(-\left(m_{1}\left(t_{i}\right)+m_{2}\left(t_{i}\right)-m_{3}\left(t_{i}\right)\right)\right) \exp \left(m_{1}\left(t_{i-1}\right)+m_{2}\left(t_{i-1}\right)-m_{3}\left(t_{i-1}\right)\right) \\
& \cdot\left(\lambda_{1}\left(t_{i}\right)+\lambda_{2}\left(t_{i}\right)-\frac{d m_{3}\left(t_{i}\right)}{d t_{i}}\right),
\end{aligned}
$$

mas,

$$
\frac{d m_{3}\left(t_{i}\right)}{d t_{i}}=\lambda_{3}\left(t_{i}\right)
$$

e assim concluímos o resultado. 
Teorema 4.2.5 Se $\left(M_{1}(t), M_{2}(t)\right)$ é um processo PPNH bivariado, dado por (4.1), então a função de verossimilhança é dada por:

$$
L(\underline{\beta})=\left[\prod_{i=1}^{n}\left(\lambda_{1}\left(t_{i}\right)+\lambda_{2}\left(t_{i}\right)-\lambda_{3}\left(t_{i}\right)\right)\right] \exp \left(-\left(m_{1}(t)+m_{2}(t)-m_{3}(t)\right)\right) .
$$

\section{Prova}

A função de verossimilhança não-condicional para $\mathcal{D}_{t}$ é dada por:

$$
L(\underline{\beta})=\left[\prod_{i=1}^{n} f\left(t_{i} \mid t_{i-1}\right)\right] P\left[T_{n+1}>t \mid T_{n}=t_{n}\right]
$$

Esta função de verossimilhança tem sido desenvolvida em muitos textos, veja por exemplo, Musa, Ianino e Okumoto, 1987.

Usando a equação (4.13) do Lema 4.2 .4 obtemos,

$$
\begin{aligned}
\prod_{i=1}^{n} f\left(t_{i} \mid t_{i-1}\right)= & {\left[\prod_{i=1}^{n}\left(\lambda_{1}\left(t_{i}\right)+\lambda_{2}\left(t_{i}\right)-\lambda_{3}\left(t_{i}\right)\right)\right] } \\
& \cdot \exp \left(-\left(m_{1}\left(t_{n}\right)+m_{2}\left(t_{n}\right)-m_{3}\left(t_{n}\right)\right)\right) .
\end{aligned}
$$

Substituindo a equação (4.12) do Lema 4.2 .3 e a equação (4.16) na equação (4.15) obtemos o resultado desejado.

Corolário 4.2.6 Se $\left(M_{1}(t), M_{2}(t)\right)$ é um processo PPNH bivariado, dado por (4.1), então a função intensidade da superposição dos processos $M_{1}(t)$ e $M_{2}(t)$ é dada por:

$$
\lambda(t)=\lambda_{1}(t)+\lambda_{2}(t)-\lambda_{3}(t)
$$

Observamos que se $\rho(t)=0 \quad \forall t \geq 0$, a função intensidade da superposição dos processos PPNH é a soma das funções intensidades dos processos componentes. 


\subsection{Inferência Bayesiana}

\subsubsection{Estimação da função de confiabilidade}

Usando o Teorema 4.2 .5 a distribuição a posteriori de $\underline{\beta}$ é dada por:

$$
\begin{aligned}
\pi\left(\underline{\beta} \mid \mathcal{D}_{t}\right) \propto & {\left[\prod_{i=1}^{n}\left(\lambda_{1}\left(t_{i}\right)+\lambda_{2}\left(t_{i}\right)-\lambda_{3}\left(t_{i}\right)\right)\right] } \\
& \cdot \exp \left(-\left(m_{1}(t)+m_{2}(t)-m_{3}(t)\right)\right) \pi(\underline{\beta}),
\end{aligned}
$$

onde $\pi(\underline{\beta})$ é uma densidade a priori para $\underline{\beta}$.

Obtemos as distribuições marginais de $\underline{\beta}$ usando o algoritmo de Gibbs com MetropolisHastings incluindo variáveis latentes.

A preditiva da função de confiabilidade a uma distância $t$ de $t_{n}$, dados os dados é dada por,

$$
E\left[S(t) \mid \mathcal{D}_{t}\right]=\int S\left(t \mid \mathcal{D}_{t}, \underline{\beta}\right) \pi\left(\underline{\beta} \mid \mathcal{D}_{t}\right) d \underline{\beta}
$$

onde

$$
S\left(t \mid \mathcal{D}_{t}, \underline{\beta}\right)=\exp \left(-\left(m\left(t_{n}+t\right)-m\left(t_{n}\right)\right)\right) V\left(t, t_{n}, \underline{\beta}\right)
$$

e

$$
m=m_{1}+m_{2} \quad V\left(t, t_{n}, \underline{\beta}\right)=\exp \left(m_{3}\left(t_{n}+t\right)-m_{3}\left(t_{n}\right)\right) .
$$

Observamos que $V\left(t, t_{n}, \underline{\beta}\right)$ é o fator introduzido devido à consideração da dependência dos processos PPNH $M_{1}(t)$ e $M_{2}(t)$ Para $\rho(t)=0 \quad \forall t \geq 0$, desde (4.20) temos que $V\left(t, t_{n}, \underline{\beta}\right)=1 \quad \forall t \geq 0$. Assim, (4.18), (4.19) e (4.20) reproduzem o caso particular, dado por Yang T.Y.,1994. Para avaliar (4.18) usamos nossa amostra de Gibbs $\underline{\beta}^{(s)}, \quad s=1, \cdots, S$ e obtemos o estimador de Monte Carlo dado por,

$$
\widehat{E}\left[S(t) \mid \mathcal{D}_{t}\right]=\widehat{S}\left(t \mid \underline{\beta}, \mathcal{D}_{t}\right)=\frac{1}{S} \sum_{s=1}^{S} S\left(t \mid \underline{\beta}^{(s)}, \mathcal{D}_{t}\right) .
$$




\subsection{Exemplo}

Consideramos um processo de Poisson bivariado $\left(M_{1}(t), M_{2}(t)\right)$, dado por (4.1), tal que:

$$
\lambda_{1}^{*}(t)=\beta_{3} t \quad \lambda_{2}^{*}(t)=\beta_{4} t^{2} \quad \lambda_{3}(t)=\frac{\beta_{1}}{\beta_{2}+t},
$$

ou seja, as funções intensidades para $M_{1}(t)$ e $M_{2}(t)$ são, respectivamente,

$$
\lambda_{1}(t)=\frac{\beta_{1}}{\beta_{2}+t}+\beta_{3} t \quad \lambda_{2}(t)=\frac{\beta_{1}}{\beta_{2}+t}+\beta_{4} t^{2} .
$$

Assim, temos que as respectivas funções de valor médio para $X(t), Y(t)$ e $U(t)$, são as seguintes,

$$
m_{1}^{*}(t)=\frac{\beta_{3} t^{2}}{2} \quad m_{2}^{*}(t)=\frac{\beta_{4} t^{3}}{3} \quad m_{3}(t)=\beta_{1} \log \left(1+\frac{t}{\beta_{2}}\right) .
$$

Logo, as respectivas funções de valor médio para $M_{1}(t)$ e $M_{2}(t)$ são as seguintes,

$$
m_{1}(t)=\beta_{1} \log \left(1+\frac{t}{\beta_{2}}\right)+\frac{\beta_{3} t^{2}}{2} \quad m_{2}(t)=\beta_{1} \log \left(1+\frac{t}{\beta_{2}}\right)+\frac{\beta_{4} t^{3}}{3} .
$$

Também podemos concluir que a função intensidade do processo superposição de $M_{1}(t)$ e $M_{2}(t)$ é,

$$
\lambda(t)=\frac{\beta_{1}}{\beta_{2}+t}+\beta_{3} t+\beta_{4} t^{2},
$$

e a função de valor médio do processo superposição de $M_{1}(t)$ e $M_{2}(t)$ é,

$$
m(t)=\beta_{1} \log \left(1+\frac{t}{\beta_{2}}\right)+\frac{\beta_{3} t^{2}}{2}+\frac{\beta_{4} t^{3}}{3} .
$$


Assim, a função de verossimilhança para o modelo de superposição dos processos dependentes $M_{1}(t)$ e $M_{2}(t)$ é a seguinte,

$$
\begin{aligned}
L\left(\beta_{1}, \beta_{2}, \beta_{3}, \beta_{4} \mid \mathcal{D}_{t}\right)= & {\left[\prod_{i=1}^{n}\left(\frac{\beta_{1}}{\beta_{2}+t_{i}}+\beta_{3} t_{i}+\beta_{4} t_{i}^{2}\right)\right] } \\
& \cdot \exp \left(-\left(\beta_{1} \log \left(1+\frac{t(n)}{\beta_{2}}\right)+\frac{\beta_{3} t(n)^{2}}{2}+\frac{\beta_{4} t(n)^{3}}{3}\right)\right) .
\end{aligned}
$$

Assumimos as seguintes distribuições a priori independentes,

$$
\beta_{i} \sim \Gamma\left(a_{i}, b_{i}\right) ; \quad a_{i}, b_{i} \quad \text { conhecidas } \quad i=1,2,3,4 .
$$

Consideramos as variáveis latentes $I_{i 1}$, definidas como na seção 2.2 , ou seja,

$$
I_{i 1} \sim \operatorname{Bernoulli}\left(p_{i 1}\right)
$$

onde,

$$
p_{i 1}=\frac{\frac{\beta_{1}}{\beta_{1}+t_{i}}}{\frac{\beta_{1}}{\beta_{2}+t_{i}}+\beta_{3} t_{i}+\beta_{4} t_{i}^{2}} \quad i=1, \cdots, n
$$

Logo, a função de densidade de $I_{i 1}$ é:

$$
\pi\left(I_{i 1} \mid \beta_{1}, \beta_{2}, \beta_{3}, \beta_{4}\right)=\frac{\left(\frac{\beta_{1}}{\beta_{2}+t_{i}}\right)^{I_{i 1}}\left(\beta_{3} t_{i}+\beta_{4} t_{i}^{2}\right)^{1-I_{i 1}}}{\frac{\beta_{1}}{\beta_{2}+t_{i}}+\beta_{3} t_{i}+\beta_{4} t_{i}^{2}} \quad i=1, \cdots, n .
$$

e a função de densidade conjunta dos $I_{i 1}$ é:

$$
\prod_{1}^{n} \pi\left(I_{i 1} \mid \beta_{1}, \beta_{2}, \beta_{3}, \beta_{4}\right)=\frac{\beta_{1}^{\sum_{i=1}^{n} I_{i 1}}\left[\prod_{i=1}^{n}\left(\beta_{3} t_{i}+\beta_{4} t_{i}^{2}\right)^{1-I_{i 1}}\right]}{\left[\prod_{i=1}^{n}\left(\frac{\beta_{1}}{\beta_{2}+t_{i}}+\beta_{3} t_{i}+\beta_{4} t_{i}^{2}\right)\right]\left[\prod_{i=1}^{n}\left(\beta_{2}+t_{i}\right)^{\left.I_{i 1}\right]}\right.} .
$$

Assim, usando (4.22), (4.23) e (4.25) temos que a distribuição conjunta a posteriori é dada por, $\pi\left(\beta_{1}, \beta_{2}, \beta_{3}, \beta_{4}, I_{i 1} \mid \mathcal{D}_{t}\right) \propto L\left(\beta_{1}, \beta_{2}, \beta_{3}, \beta_{4} \mid \mathcal{D}_{t}\right)\left[\prod_{j=1}^{4} \beta_{j}^{a_{j}-1} e^{-b_{j} \beta_{j}}\right]\left[\prod_{i=1}^{n} \pi\left(I_{i 1} \mid \beta_{1}, \beta_{2}, \beta_{3}, \beta_{4}\right)\right]$. 
Portanto, o algoritmo de Metropolis-Hastings é dado pelas seguintes etapas:

1. Construir $I_{i 1} \quad i=1, \cdots, n$ com distribuições Bernoulli com parâmetros $p_{i 1}$ dados pelas equações (4.24).

2. Gerar $\beta_{1}$ tal que:

$$
\beta_{1} \mid \beta_{2}, \beta_{3}, \beta_{4}, I_{i 1}, \mathcal{D}_{t} \sim \Gamma\left(a_{1}+\sum_{i=1}^{n} I_{i 1}, b_{1}+\log \left(1+\frac{t_{n}}{\beta_{2}}\right)\right) .
$$

3. Gerar $\beta_{2}$ tal que,

$$
\beta_{2} \mid \beta_{1}, \beta_{3}, \beta_{4}, I_{i 1}, \mathcal{D}_{t} \sim \Gamma\left(a_{2}, b_{2}\right) \psi_{1}\left(\beta_{1}, \beta_{2}, \beta_{3}, \beta_{4}\right)
$$

onde,

$$
\psi_{1}\left(\beta_{1}, \beta_{2}, \beta_{3}, \beta_{4}\right)=\exp \left(-\beta_{1} \log \left(1+\frac{t_{n}}{\beta_{2}}\right)-\sum_{i=1}^{n} I_{i 1} \log \left(\beta_{2}+t_{i}\right)\right) .
$$

4. Gerar $\beta_{3}$ tal que,

$$
\left.\beta_{3} \mid \beta_{1}, \beta_{2}, \beta_{4}\right), I_{i 1}, \mathcal{D}_{t} \sim \Gamma\left(a_{3}, b_{3}\right) \psi_{2}\left(\beta_{1}, \beta_{2}, \beta_{3}, \beta_{4}\right)
$$

onde:

$$
\psi_{2}\left(\beta_{1}, \beta_{2}, \beta_{3}, \beta_{4}\right)=\exp \left(-\frac{\beta_{3} t_{n}^{2}}{2}-\sum_{i=1}^{n}\left(1-I_{i 1}\right) \log \left(\beta_{3} t_{i}+\beta_{4} t_{i}^{2}\right)\right) .
$$

5. Gerar $\beta_{4}$ tal que,

$$
\left.\beta_{4} \mid \beta_{1}, \beta_{2}, \beta_{3}\right), I_{i 1}, \mathcal{D}_{t} \sim \Gamma\left(a_{4}, b_{4}\right) \psi_{3}\left(\beta_{1}, \beta_{2}, \beta_{3}, \beta_{4}\right),
$$

onde,

$$
\psi_{3}\left(\beta_{1}, \beta_{2}, \beta_{3}, \beta_{4}\right)=\exp \left(-\frac{\beta_{4} t_{n}^{3}}{3}-\sum_{i=1}^{n}\left(1-I_{i 1}\right) \log \left(\beta_{3} t_{i}+\beta_{4} t_{i}^{2}\right)\right) .
$$




\subsection{Modelagem Alternativa}

Uma dificuldade que surge ao considerar a abordagem estudada até agora neste capítulo, é que não é possível observar o processo $U(t)$, que causa a dependência entre $M_{1}(t)$ e $M_{2}(t)$. Ou seja, não conhecemos, por exemplo, a função de valor médio $m_{3}(t)$, e portanto a covariância e o coeficiente de correlação são desconhecidos. Assim, para modelar a superposição dos processos $M_{1}(t)$ e $M_{2}(t)$ usando a função de verossimilhança dada pelo Teorema 4.2.5, temos que supor conhecidas as funções intensidades $\lambda_{1}^{*}(t), \lambda_{2}^{*}(t)$ e $\quad \lambda_{3}^{*}(t)$, ou as funções de valor médio $m_{1}^{*}(t), m_{2}^{*}(t)$ e $\quad m_{3}^{*}(t)$. Uma possível metodologia que podemos aplicar para resolver este problema é considerar diferentes formas para estas funções. Daí, obteríamos vários modelos para estudar nosso conjunto de dados. Finalmente, depois de estimar os parâmetros de cada um dos modelos, escolheríamos um modelo entre eles usando o fator de Bayes, como será explicado no capítulo seguinte.

Outra forma de resolver o problema de desconhecimento do processos comum $U(t)$, é supor o coeficiente de correlação $\rho(t)$ constante e igual a $\rho$, ou seja usando (4.3) obtemos,

$$
\rho=\rho\left(M_{1}(t), M_{2}(t)\right)=\frac{m_{3}(t)}{\left[m_{1}(t) m_{2}(t)\right]^{1 / 2}} \quad \forall t \geq 0 .
$$

Assim,

$$
m_{3}(t)=\rho\left[m_{1}(t) m_{2}(t)\right]^{1 / 2} \quad \forall t \geq 0
$$

Logo,

$$
\frac{d m_{3}(t)}{d t}=\frac{\rho\left[\lambda_{1}(t) m_{2}(t)+\lambda_{2}(t) m_{1}(t)\right]}{2\left[m_{1}(t) m_{2}(t)\right]^{1 / 2}}
$$

ou seja,

$$
\frac{d m_{3}(t)}{d t}=\frac{\rho}{2}\left(\lambda_{1}(t)\left[\frac{m_{2}(t)}{m_{1}(t)}\right]^{1 / 2}+\lambda_{2}(t)\left[\frac{m_{1}(t)}{m_{2}(t)}\right]^{1 / 2}\right) .
$$

Definindo $W(t)$ tal que,

$$
W(t)=\frac{\lambda_{1}(t)\left[\frac{m_{2}(t)}{m_{1}(t)}\right]^{1 / 2}+\lambda_{2}(t)\left[\frac{m_{1}(t)}{m_{2}(t)}\right]^{1 / 2}}{\lambda_{1}(t)+\lambda_{2}(t)},
$$


obtemos,

$$
\frac{d m_{3}(t)}{d t}=\left[\lambda_{1}(t)+\lambda_{2}(t)\right] \frac{\rho W(t)}{2} .
$$

Assim, poderíamos substituir (4.32) no Lema 4.2.4 e rescrever o Teorema 4.2.5 na seguinte forma,

Teorema 4.5.1 Se $\left(M_{1}(t), M_{2}(t)\right)$ é um processo PPNH bivariado, dado por (4.1), $e$ supondo que $\rho(t)=\rho \quad \forall t \geq 0$, então a função de verossimilhança é dada por:

$$
\begin{aligned}
L(\underline{\beta}, \rho)= & {\left[\prod_{i=1}^{n}\left(\lambda_{1}\left(t_{i}\right)+\lambda_{2}\left(t_{i}\right)\right)\left(1-\frac{\rho}{2} W\left(t_{i}\right)\right)\right] } \\
& \cdot \exp \left(-\left(m_{1}(t)+m_{2}(t)-\rho\left[m_{1}(t) m_{2}(t)\right]^{1 / 2}\right)\right)
\end{aligned}
$$

onde $W\left(t_{i}\right)$ é calculado em cada ponto de $\mathcal{D}_{t}$ usando a (4.31).

Aplicações numéricas serão desenvolvidas no capítulo 6. 


\section{Capítulo 5}

\section{Discriminação Bayesiana de modelos}

\subsection{Estimativa da verossimilhança marginal usando amostragem por importância}

A verossimilhança marginal , $P(\mathcal{D} \mid M)$, é usada para construir testes de hipóteses Bayesianos, selecionar modelos, medir a incerteza de um modelo e portanto discriminar dentro de um grupo de modelos possíveis e determinar qual deles se ajusta melhor ao conjunto de dados em estudo. Inicialmente, por simplicidade, consideramos somente um modelo. A verossimilhança marginal é definida da seguinte forma, (veja, por exemplo, B.P. Carlin e T.A. Louis,1996).

$$
P(\mathcal{D} \mid M)=\int L(\mathcal{D} \mid \underline{\beta}) p(\underline{\beta}) d \underline{\beta} .
$$

Para estimar $P(\mathcal{D} \mid M)$, podemos gerar uma amostra $\left(\underline{\beta}^{(1)}, \underline{\beta}^{(2)}, \cdots, \underline{\beta}^{(S)}\right)$ a partir da priori $p(\underline{\beta})$, e calcular uma estimativa de Monte Carlo dada por,

$$
\widehat{P}(\mathcal{D} \mid M)=\frac{1}{S} \sum_{s=1}^{S} L\left(\mathcal{D} \mid \underline{\beta}^{(s)}\right)
$$


Outra possibilidade é usar amostragem por importância para estimar $P(\mathcal{D} \mid M)$ supondo que podemos amostrar a partir de uma densidade proporcional a uma função positiva, $g(\underline{\beta})$, onde $c g(\underline{\beta})$ é uma f.d.p., ou seja:

$$
c^{-1}=\int g(\underline{\beta}) d \underline{\beta} .
$$

Logo,

$$
P(\mathcal{D} \mid M)=\int L(\mathcal{D} \mid \underline{\beta})\left[\frac{p(\underline{\beta})}{c g(\underline{\beta})}\right] c g(\underline{\beta}) d \underline{\beta} .
$$

Dada uma amostra $\left(\underline{\beta}^{(1)}, \underline{\beta}^{(2)}, \cdots, \underline{\beta}^{(s)}\right)$ obtida a partir da densidade $c g(\underline{\beta})$, temos o estimador:

$$
P_{0}(\mathcal{D} \mid M)=\frac{1}{S} \sum_{s=1}^{S}\left[\frac{L\left(\mathcal{D} \mid \underline{\beta}^{(s)}\right) p\left(\underline{\beta}^{(s)}\right)}{c g\left(\underline{\beta}^{(s)}\right)}\right] .
$$

Se não podemos determinar c, então usamos MCMC para obter uma amostra para estimá-lo. Uma estimativa de consistente em simulação é dada por,

$$
\hat{c}=\frac{1}{S} \sum_{s=1}^{S} \frac{p\left(\underline{\beta}^{(s)}\right)}{g\left(\underline{\beta}^{(s)}\right)} .
$$

Logo, o estimador geral de $P(\mathcal{D} \mid M)$, usando amostragem por importância é,

$$
P_{1}(\mathcal{D} \mid M)=\frac{P_{0}(\mathcal{D} \mid M)}{\hat{c}}
$$

ou seja,

$$
P_{1}(\mathcal{D} \mid M)=\frac{\sum_{s=1}^{S}\left[\frac{L\left(\mathcal{D} \mid \underline{\beta}^{(s)}\right) p\left(\hat{\beta}^{(s)}\right)}{g\left(\underline{\beta}^{(s)}\right)}\right]}{\sum_{s=1}^{S}\left[\frac{p\left(\underline{\beta}^{(s)}\right)}{g\left(\underline{\beta}^{(s)}\right)}\right]}
$$

$\mathrm{Se}$

$$
\omega\left(\underline{\beta}^{(s)}\right)=\frac{p\left(\underline{\beta}^{(s)}\right)}{g\left(\underline{\beta}^{(s)}\right)}
$$

podemos escrever (5.4) na forma seguinte:

$$
P_{1}(\mathcal{D} \mid M)=\frac{\sum_{s=1}^{S} L\left(\mathcal{D} \mid \underline{\beta}^{(s)}\right) \omega\left(\underline{\beta}^{(s)}\right)}{\sum_{s=1}^{S} \omega\left(\underline{\beta}^{(s)}\right)} .
$$


Podemos considerar alguns casos particulares para a função $g$ que aparece implicitamente em (5.6) devido a (5.5):

(i) $\underline{g=p}$.

Isto é, a função usada na amostragem por importância é uma f.d.p. que coincide com a priori e portanto,

$$
\omega\left(\underline{\beta}^{(s)}\right)=1 \quad \text { e } \quad \sum_{s=1}^{S} \omega\left(\underline{\beta}^{(s)}\right)=S .
$$

Assim, usando (5.6) obtemos

$$
P_{2}(\mathcal{D} \mid M)=\frac{1}{S} \sum_{s=1}^{S} L\left(\mathcal{D} \mid \underline{\beta}^{(s)}\right),
$$

que coincide com a estimativa de Monte Carlo (5.2).

(ii) $g=L p$.

Temos,

$$
\omega\left(\underline{\beta}^{(s)}\right)=\frac{p\left(\underline{\beta}^{(s)}\right)}{L\left(\mathcal{D} \mid \underline{\beta}^{(s)}\right) p\left(\underline{\beta}^{(s)}\right)}=\frac{1}{L\left(\mathcal{D} \mid \underline{\beta}^{(s)}\right)} .
$$

Logo, obtemos a estimativa

$$
P_{3}(\mathcal{D} \mid M)=\frac{S}{\sum_{s=1}^{S}\left[\frac{1}{L\left(\mathcal{D} \mid \underline{\beta}^{(\delta)}\right)}\right]}=\frac{1}{\frac{1}{S} \sum_{s=1}^{S}\left[\frac{1}{L\left(\mathcal{D} \mid \underline{\beta}^{(\delta)}\right)}\right]},
$$

ou seja, $P_{3}(\mathcal{D} \mid M)$ é a média harmônica $\operatorname{dos} L\left(\mathcal{D} \mid \underline{\beta}^{(s)}\right)$.

Observamos que, neste caso, $g$ representa a f.d.p. da distribuição a posteriori.

(iii) $\underline{g=\lambda p+(1-\lambda) L p \quad \text { com } \quad 0<\lambda<1}$

Neste caso, a função usada para amostragem por importância é uma combinação convexa de $p$ e $L p$, ou seja uma mistura das densidades a priori e a posteriori. O estimador obtido é 


$$
P_{4}(\mathcal{D} \mid M)=\frac{\sum_{s=1}^{S}\left[\frac{L\left(\mathcal{D} \mid \underline{\beta}^{(s)}\right) p\left(\underline{\beta}^{(s)}\right)}{\lambda p\left(\underline{\beta}^{(s)}\right)+(1-\lambda) L\left(\mathcal{D} \mid \underline{\beta}^{(s)}\right) p\left(\underline{\beta}^{(s)}\right)}\right]}{\sum_{s=1}^{S}\left[\frac{p\left(\underline{\beta}^{(s)}\right)}{\lambda p\left(\underline{\beta}^{(s)}\right)+(1-\lambda) L\left(\mathcal{D} \mid \underline{\beta}^{(s)}\right) p\left(\underline{\beta}^{(s)}\right)}\right]}
$$

Vejamos agora quais são as principais vantagens e desvantagens destas estimativas. Em relação a. $P_{2}$, ocorre que se a verossimilhança marginal é apontada comparada com a priori, então $P_{2}$ será um estimador muito ineficiente, no sentido de que a maioria dos termos na soma terão valores muito pequenos e assim na prática o processo computacional fornecerá valores zeros para estes termos, ocorrendo em alguns casos que o valor computacional de $P_{2}$ seja zero, e em outros casos que o valor de $P_{2}$ está fortemente influenciado por uns poucos valores da verossimilhança. Isto é indicado na teoria pelo fato de que a convergência de $P_{2}$ em lei a uma distribuição normal é lenta.

Por outro lado, o estimador $P_{3}$, dado por (5.9), converge quase certamente ao valor exato, $P(\mathcal{D} \mid M)$ quando $S \longrightarrow \infty$. Porém, em geral não satisfaz um Teorema do Limite Central Gaussiano e sua variância muitas vezes tende para o infinito, (veja, A.E. Raftery, 1996).

Tentamos evitar os problemas que acabamos de comentar, ao considerarmos $P_{4}$, dado por (5.10). Assim, conseguimos evitar a instabilidade de $P_{3}$ e além disso satisfazer um teorema do limite central gaussiano. A desvantagem é que para calcular a estimativa $P_{4}$ devemos simular tanto da priori quanto da posteriori.

\subsection{Fator de Bayes}

A seção anterior foi dedicada ao estudo de estimativas da verossimilhança marginal considerando somente um modelo. Agora veremos o que ocorre quando devemos escolher entre vários modelos.

Se considerarmos os modelos $M_{1}$ e $M_{2}$, o fator de Bayes é a razão das verosimilhanças 
marginais observadas para os modelos, ou seja:

$$
B_{12}=\frac{P\left(\mathcal{D} \mid M_{1}\right)}{P\left(\mathcal{D} \mid M_{2}\right)}
$$

Se tivermos vários modelos, $M_{1}, M_{2}, \cdots, M_{k}$, podemos fazer a escolha considerando os modelos dois a dois, usando (5.11) e a seguinte

\section{Definição}

$$
M_{i} \preceq M_{j} \Longleftrightarrow B_{i j}<1
$$

onde, $M_{i} \preceq M_{j}$ é lido: o modelo j é preferível ao modelo i.

Assim, obtemos a seguinte ordenação de todos os modelos em estudo:

$$
M_{(1)} \preceq M_{(2)} \preceq \cdots \preceq M_{(k)},
$$

onde $((1),(2), \cdots,(k))$ é uma permutação de $(1,2, \cdots, k)$.

Logo, se não tivermos outras restrições adicionais, escolheríamos o modelo $M_{(k)}$ e, se tivermos essas restrições, escolheríamos o modelo $M_{(j)}$, tal que

$$
M_{(1)} \preceq \cdots \preceq M_{(j-1)} \preceq M_{(j)} \preceq M_{(j+1)} \preceq \cdots \preceq M_{(k)},
$$

onde $M_{(j+1)}, \cdots, M_{(k)}$ não satisfazem essas restrições adicionais. 


\section{Capítulo 6}

\section{Aplicações numéricas}

\subsection{Exemplo para o Capítulo 2}

\begin{tabular}{cccccccc}
\hline \hline$t_{i}$ & $x_{i}$ & $t_{i}$ & $x_{i}$ & $t_{i}$ & $x_{i}$ & $t_{i}$ & $x_{i}$ \\
\hline \hline 10.17458 & 2 & 220.21197 & 2 & 408.41503 & 3 & 549.74908 & 3 \\
10.37464 & 2 & 268.24415 & 2 & 413.01906 & 3 & 587.17665 & 2 \\
15.53894 & 3 & 274.58956 & 2 & 418.10782 & 2 & 638.99179 & 3 \\
58.18520 & 2 & 281.26981 & 2 & 422.57861 & 2 & 653.49720 & 2 \\
73.94862 & 3 & 281.70729 & 3 & 431.64756 & 3 & 660.25647 & 2 \\
97.26943 & 2 & 311.38187 & 3 & 434.52618 & 3 & 690.52829 & 2 \\
106.21964 & 2 & 315.88718 & 3 & 441.14613 & 3 & 711.52416 & 2 \\
151.81222 & 3 & 325.34200 & 2 & 443.26516 & 3 & 722.77106 & 3 \\
168.67324 & 2 & 326.23853 & 2 & 480.22311 & 2 & 761.51431 & 3 \\
198.77185 & 3 & 327.14524 & 3 & 5334.39471 & 3 & 771.60783 & 3 \\
\hline \hline
\end{tabular}

Tabela 6.1 . Dados gerados.

Os dados da tabela 6.1 foram simulados a partir de um processo PPNH, com função intensidade 


$$
\lambda\left(t_{i}\right)=\left(\frac{e^{\gamma+\tau x_{i}}}{1+e^{\gamma+\tau x_{i}}}\right)\left(\frac{\alpha}{\beta_{1}+t_{i}}\right) e^{-\xi_{1} x_{i}}+\left(\frac{1}{1+e^{\gamma+\tau x_{i}}}\right)\left(\beta_{2} t_{i}\right) e^{-\xi_{2} x_{i}}
$$

para os valores $\alpha=20, \beta_{1}=100, \beta_{2}=0.0005, \gamma=-1.0, \tau=2, \xi_{1}=0.75$ e $\xi_{2}=-0.75 ; 40$ observações foram geradas considerando a covariada $x$ com valores 2 e 3 (20 observações para cada nível da covariada $x$ ).

Para uma análise Bayesiana dos dados de software da Tabela 6.1, estamos supondo uma superposição dos processos Musa e Okumoto com o processo exponencial, considerando os três modelos mencionados no capítulo 2 .

\subsubsection{Modelo 1: Superposição dos processos Musa e Okumoto com o processo Exponencial}

Escolhemos as seguintes distribuições a priori,

$$
\begin{aligned}
\alpha & \sim \Gamma(400,20) \\
\beta_{1} & \sim \Gamma(2500,25) \\
\beta_{2} & \sim \Gamma(0.5,1000) .
\end{aligned}
$$

Na tabela 6.2, apresentamos resumos a posteriori correspondentes aos parâmetros $\alpha, \beta_{1}$ e $\beta_{2}$.

\begin{tabular}{ccccc}
\hline \hline & & \multicolumn{4}{c}{ Intervalo de } \\
Parâmetro & Média & D.P. & Credibilidade (95\%) & $\widehat{R}$ \\
\hline \hline$\alpha$ & 19.48811 & 0.89316 & $(17.69677 ; 21.22156)$ & 1.00 \\
$\beta_{1}$ & 100.06478 & 2.02220 & $(96.05514 ; 103.97208)$ & 1.00 \\
$\beta_{2}$ & 0.000028678 & 0.00017499 & $(0.00000055447 ; 0.000066897)$ & 1.00 \\
\hline \hline
\end{tabular}

Tabela 6.2. Resumos a posteriori para o modelo 1 . 
Os resultados da tabela 2 foram obtidos gerando uma amostra de Gibbs de tamanho 1000, da forma indicada no capítulo 1 . Também incluímos nesta tabela o fator de redução de escala $\hat{R}$ (veja, Gelman e Rubin, 1992). Como $\sqrt{\widehat{R}}<1.1$ para todos os parâmetros, observamos a convergência do algoritmo de amostrador de Gibbs.

\subsubsection{Modelo 2: Superposição dos processos Musa e Okumoto com o processo Exponencial incluindo uma covariada que afeta somente as probabilidades de incidência.}

Escolhemos as seguintes distribuições a priori,

$$
\begin{aligned}
\alpha & \sim \Gamma(400,20) \\
\beta_{1} & \sim \Gamma(2500,25) \\
\beta_{2} & \sim \Gamma(0.5,1000) \\
\gamma & \sim N(-1,0.5) \\
\tau & \sim N(2,0.2) .
\end{aligned}
$$

A partir das distribuições condicionais para $I, \alpha, \beta_{1}, \beta_{2}, \gamma$ e $\tau$ dadas em (2.21), geramos 10 cadeias de Gibbs separadas, obtendo finalmente uma amostra de tamanho 1000.

$\mathrm{Na}$ tabela 6.3, apresentamos os resumos a posteriori correspondentes aos parâmetros $\alpha, \beta_{1}, \beta_{2}, \gamma$ e $\tau$, e na figura 6.1, temos gráficos das densidades marginais a posteriori, considerando a amostra obtida. Também, incluímos estimações de $\widehat{R}$ (veja, Gelman e Rubin, 1992) para todos os parâmetros, para verificar a convergência, $(\sqrt{\widehat{R}}<1.1$ para todos os parâmetros ). 


\begin{tabular}{ccccc}
\hline \hline & & \multicolumn{3}{c}{ Intervalo de } \\
Parâmetro & Média & D.P. & Credibilidade (95\%) & $\widehat{R}$ \\
\hline \hline$\alpha$ & 19.32561 & 0.95725 & $(17.48068 ; 21.32249)$ & 1.01 \\
$\beta_{1}$ & 100.02738 & 2.03564 & $(96.40102 ; 103.89391)$ & 1.00 \\
$\beta_{2}$ & 0.0067222 & 0.00309 & $(0.00090326 ; 0.013309)$ & 1.01 \\
$\gamma$ & -0.55449 & 0.48432 & $(-1.51179 ; 0.37983)$ & 1.01 \\
$\tau$ & 2.22507 & 0.18535 & $(1.87137 ; 2.59640)$ & 1.01 \\
\hline \hline
\end{tabular}

Tabela 6.3. Resumos a posteriori para o modelo 2.
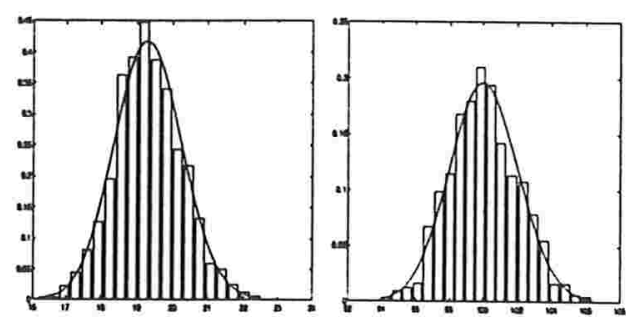

$\beta_{1}$

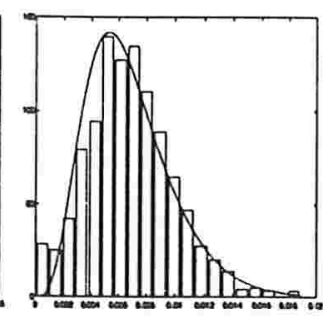

$\beta_{2}$

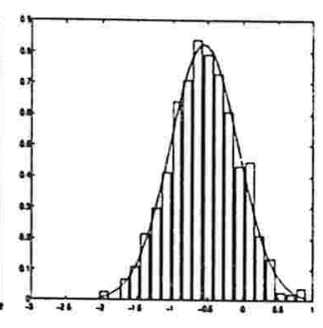

$\gamma$

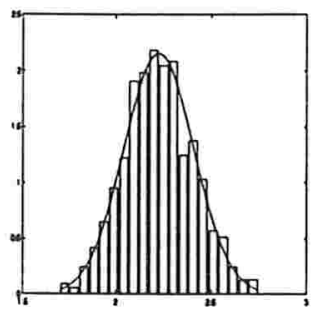

$\tau$

Figura 6.1. Densidades marginais a posteriori aproximadas para o modelo 2

6.1.3 Modelo 3: Superposição dos processos Musa e Okumoto com o processo Exponencial incluindo uma covariada que influencia as probabilidades de incidência e as funções intensidades.

Escolhemos as seguintes distribuições a priori,

$$
\begin{aligned}
\alpha & \sim \Gamma(400,20) \\
\beta_{1} & \sim \Gamma(2500,25) \\
\beta_{2} & \sim \Gamma(0.5,1000)
\end{aligned}
$$




$$
\begin{aligned}
\gamma & \sim N(-1,0.5) \\
\tau & \sim N(2,0.2) \\
\xi_{1} & \sim N(0.75 ; 0.5) \\
\xi_{2} & \sim N(-0.75 ; 0.2) .
\end{aligned}
$$

Geramos 10 cadeias separadas de Gibbs, a partir das distribuições condicionais, cada uma com 2200 iterações. Eliminamos os primeiros 200 elementos de cada cadeia e logo selecionamos um de cada 10 elementos. Obtivemos assim uma amostra final de tamanho 2000 para cada parâmetro .

$\mathrm{Na}$ tabela 6.4, apresentamos resumos a posteriori para os parâmetros $\alpha, \beta_{1}, \beta_{2}, \gamma, \tau, \xi_{1}$ e $\xi_{2}$. Para verificar a convergência, incluímos estimativas para os $R$ correspondentes ao método de Gelman e Rubin.

\begin{tabular}{ccccc}
\hline \hline & & \multicolumn{3}{c}{ Intervalo de } \\
Parâmetro & Média & D.P. & Credibilidade (95\%) & $\widehat{R}$ \\
\hline \hline$\alpha$ & 20.10435 & 0.99830 & $(18.24249 ; 22.04476)$ & 1.00 \\
$\beta_{1}$ & 99.99260 & 1.97943 & $(96.09743 ; 103.93848)$ & 1.00 \\
$\beta_{2}$ & 0.0055428 & 0.0029064 & $(0.0015149 ; 0.012550)$ & 1.05 \\
$\gamma$ & -0.54008 & 0.52149 & $(-1.51051 ; 0.58589)$ & 1.07 \\
$\tau$ & 2.28826 & 0.18152 & $(1.93348 ; 2.63714)$ & 1.06 \\
$\xi_{1}$ & 1.36157 & 0.42651 & $(0.58229 ; 2.26105)$ & 1.00 \\
$\xi_{2}$ & -0.82256 & 0.18874 & $(-1.20223 ;-0.45599)$ & 1.05 \\
\hline \hline
\end{tabular}

Tabela 6.4. Resumos a posteriori para o modelo 3 .

$\mathrm{Na}$ figura 6.2, incluímos os gráficos das densidades marginais a posteriori aproximadas para todos os parâmetros $\alpha, \beta_{1}, \beta_{2}, \gamma, \tau, \xi_{1}$ e $\xi_{2}$. 


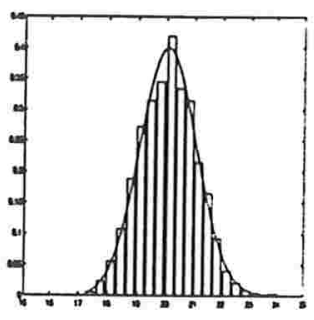

$\alpha$

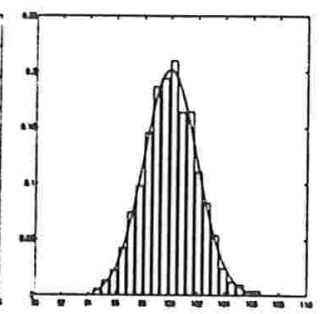

$\beta_{1}$

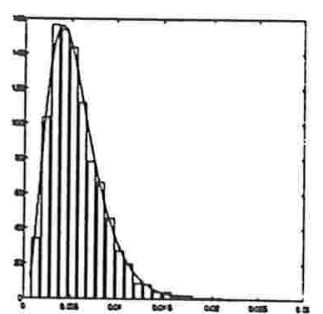

$\beta_{2}$

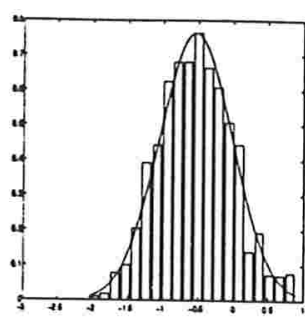

$\gamma$

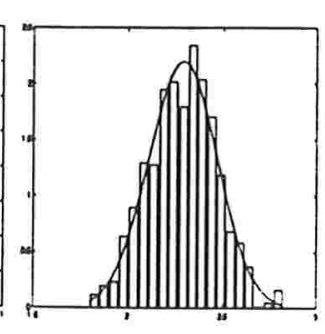

$\tau$

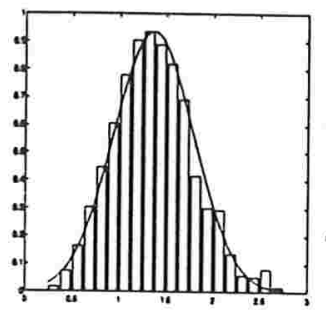

$\xi_{1}$

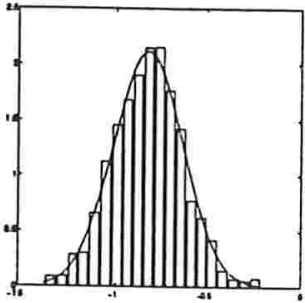

$\xi_{2}$

Figura 6.2. Densidades marginais a posteriori aproximadas para o modelo 3 .

Enquanto à seleção de modelos, estimamos a função de verossimilhança marginal para cada modelo considerado. Os valores destas estimativas são dadas na Tabela 6.5.

\begin{tabular}{lc}
\hline \hline Modelo & Verossimilhança marginal estimada \\
\hline \hline Modelo 1 & $8.748 \cdot 10^{-75}$ \\
\hline Modelo 2 & $4.692 \cdot 10^{-71}$ \\
\hline Modelo 3 & $8.204 \cdot 10^{-8}$ \\
\hline \hline
\end{tabular}

Tabela 6.5. Valores estimados para a funções de verossimilhança marginais.

Na tabela 6.5, temos os valores estimados para as funções de verossimilhança marginais calculados de acordo com a equação (5.2), no capítulo 5. A partir dos resultados da tabela 6.5 , podemos ordenar os modelos sob estudo da seguinte forma,

$$
M_{1} \preceq M_{2} \preceq M_{3},
$$

e assim, concluir que o melhor modelo para ajustar os dados da tabela 6.1 é o modelo 3 . 


\subsection{Exemplo para o Capítulo 3}

Simulamos os dados da Tabela 6.6 a partir de um processo PPNH com função intensidade Weibull-exponenciada, considerando os seguintes valores para os parâmetros: $\alpha=1.2, \theta=0.75, \quad e \quad \sigma=100$; geramos no total, 36 observações.

\begin{tabular}{cccccc}
\hline \hline $\mathrm{i}$ & $t_{i}$ & $\mathrm{i}$ & $t_{i}$ & $\mathrm{i}$ & $t_{i}$ \\
\hline \hline 1 & 1.26835 & 13 & 656.16081 & 25 & 980.52727 \\
2 & 15.72966 & 14 & 671.52321 & 26 & 1062.16707 \\
3 & 32.23801 & 15 & 719.57745 & 27 & 1066.97822 \\
4 & 110.41090 & 16 & 780.01486 & 28 & 1092.42460 \\
5 & 113.40610 & 17 & 811.17553 & 29 & 1093.95373 \\
6 & 200.98991 & 18 & 826.83801 & 30 & 1136.13604 \\
7 & 221.12769 & 19 & 831.33000 & 31 & 1147.00027 \\
8 & 490.28306 & 20 & 886.65706 & 32 & 1340.85381 \\
9 & 499.84578 & 21 & 887.42836 & 33 & 1382.16824 \\
10 & 554.87830 & 22 & 917.09363 & 34 & 1410.87073 \\
11 & 565.07198 & 23 & 921.82979 & 35 & 1414.14548 \\
12 & 639.51697 & 24 & 968.73424 & 36 & 1560.62059 \\
\hline \hline
\end{tabular}

Tabela 6.6. Dados gerados a partir de um processo Weibull-exponencial com $\alpha=1.2, \theta=0.75, \sigma=100$

Para uma análise Bayesiana dos dados de confiabilidade de software da Tabela 6.6, assumimos um processo do tipo NHPP-II com uma função intensidade Weibullexponenciada com distribuições a priori dadas por,

$$
\begin{aligned}
\alpha & \sim \Gamma(7.2 ; 6) \\
\sigma & \sim \Gamma(1000 ; 10) \\
\theta & \sim \operatorname{Beta}(1500 ; 500) .
\end{aligned}
$$


Neste caso particular consideramos mais adequado escolher uma distribuição a priori Beta para $\theta$, desde que a forma de banheira que estamos considerando ocorre quando $0<\theta<1$.

A partir das distribuições condicionais para $\alpha, \theta$ e $\sigma$ dadas em (3.6), geramos 10 cadeias Gibbs separadas, cada uma das quais com ciclos de até 1200 iterações. Para cada cadeia, descartamos os primeiros 200 elementos ( período de aquecimento ). Monitoramos a convergência das amostras de Gibbs usando o método de Gelman e Rubin, 1992, baseado na técnica de análise de variância para determinar se precisamos de mais iterações . Para cada parâmetro, consideramos um elemento da amostra em cada dez e assim obtemos uma amostra de tamanho 1000 .

Na Tabela 6.7, temos os resumos a posteriori para os parâmetros $\alpha, \theta$ e $\sigma$. Também, incluímos a estimativa $\widehat{R}$, da redução da escala potencial para cada um dos parâmetros. Observamos convergência para todos os parâmetros.

\begin{tabular}{ccccc}
\hline \hline & & \multicolumn{3}{c}{ Intervalo de } \\
Parâmetro & Média & D.P. & Credibilidade (95\%) & $\hat{R}$ \\
\hline \hline$\alpha$ & 1.23568 & 0.05022 & $(1.12168 ; 1.31881)$ & 1.008 \\
$\theta$ & 0.74978 & 0.00911 & $(0.73282 ; 0.76774)$ & 1.007 \\
$\sigma$ & 99.59496 & 3.20429 & $(93.69764 ; 106.24767)$ & 1.000 \\
\hline \hline
\end{tabular}

Tabela 6.7. Resumos a posteriori para o processo Weibull-exponencial.

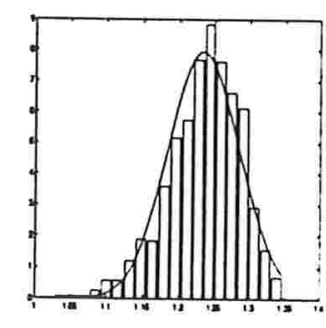

alfa

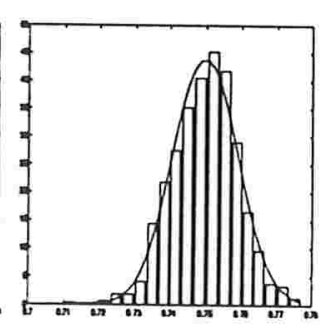

teta

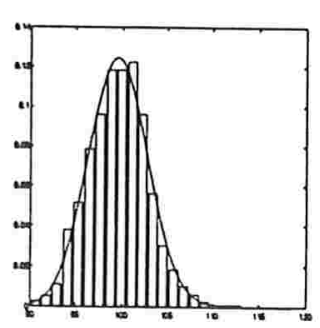

sigma

Figura 6.3. Densidades marginais a posteriori aproximadas ( processo com função intensidade Weibull-exponenciada ). 
Na figura 6.3, apresentamos os gráficos das densidades marginais aproximadas, considerando $S=1000$ amostras de Gibbs.

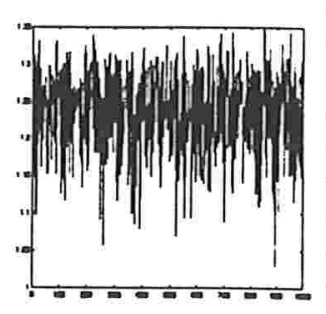

alfa

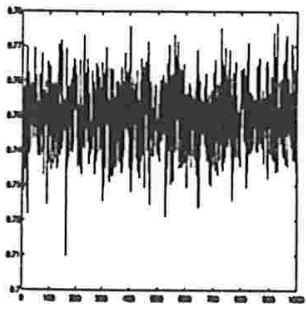

teta

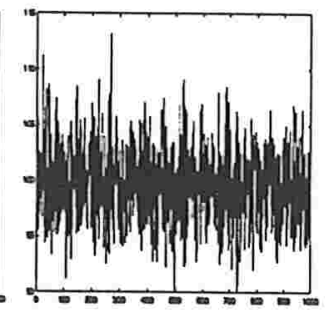

sigma

Figura 6.4. Convergência do algoritmo de Gibbs para o processo Weibull-exponencial.

Na figura 6.4, temos gráficos mostrando a convergência do algoritmo Gibbs para o processo Weibull-exponencial. Observamos convergência para todos os parâmetros.

Agora, vamos considerar o mesmo conjunto de dados e a superposição de um processo Musa e Okumoto com um processo exponencial com função intensidade dada por (2.6). Para uma análise Bayesiana consideramos as distribuições a priori seguintes:

$$
\begin{aligned}
\alpha & \sim \Gamma(380 ; 20) \\
\beta_{1} & \sim \Gamma(5000 ; 50) \\
\beta_{2} & \sim \Gamma(0.03 ; 10000) .
\end{aligned}
$$

A partir das distribuições condicionais para $I, \alpha, \beta_{1}$ e $\beta_{2}$ dadas em (2.13) geramos 10 cadeias Gibbs separadas, cada uma delas com ciclos de até 1200 iterações. Eliminamos os primeiros 200 elementos de cada cadeia, e selecionamos um de cada 10 elementos de cada cadeia. Obtemos assim uma amostra de tamanho 1000 para cada parâmetro.

Na Tabela 6.8, apresentamos os resumos a posteriori dos parâmetros $\alpha, \beta_{1}$ e $\beta_{2}$. Também na Tabela 6.8 apresentamos a estimativa $\widehat{R}$, de Gelman e Rubin para verificar convergência dos parâmetros. 


\begin{tabular}{ccccc}
\hline \hline \multirow{2}{*}{ Parâmetro } & Média & D.P. & Credibilidade (95\%) & $\widehat{R}$ \\
\hline \hline$\alpha$ & 18.93163 & 0.90263 & $(17.14557 ; 20.72774)$ & 1.004 \\
$\beta_{1}$ & 100.17290 & 2.04359 & $(96.00419 ; 104.14977)$ & 1.000 \\
$\beta_{2}$ & 0.0000045830 & 0.0000035707 & $(0.000000017253 ; 0.000013042)$ & 0.998 \\
\hline \hline
\end{tabular}

Tabela 6.8. Resumos a posteriori para a superposição dos processos Musa-Okumoto com exponencial

Finalmente, consideramos a superposição do processo Musa-Okumoto com o processo exponencial, levando em conta as probabilidades de incidência, ou seja usamos a função intensidade dada por (3.8). Assumimos as seguintes distribuições a priori para $\alpha, \beta_{1}, \beta_{2}$ e $\theta$

$$
\begin{aligned}
\alpha & \sim \Gamma(500 ; 20) \\
\beta_{1} & \sim \Gamma(2500 ; 25) \\
\beta_{2} & \sim \Gamma(0.25 ; 1000) \\
\theta & \sim \operatorname{Beta}(0.48 ; 0.12) .
\end{aligned}
$$

\begin{tabular}{ccccc}
\hline \hline & & \multicolumn{4}{c}{ Intervalo de } \\
Parâmetro & Média & D.P. & Credibilidade $(95 \%)$ & $\widehat{R}$ \\
\hline \hline$\alpha$ & 24.99587 & 1.10233 & $(22.85377 ; 27.24914)$ & 1.007 \\
$\beta_{1}$ & 100.00332 & 2.12828 & $(95.76092 ; 104.074989)$ & 1.003 \\
$\beta_{2}$ & 0.000023029 & 0.0000057885 & $(0.000012754 ; 0.000035539)$ & 1.004 \\
$\theta$ & 0.20585 & 0.079171 & $(0.073939 ; 0.38613)$ & 1.042 \\
\hline \hline
\end{tabular}

Tabela 6.9. Resumos a posteriori para a superposição dos processos Musa e Okumoto com exponencial, considerando a probabilidade de incidência. 


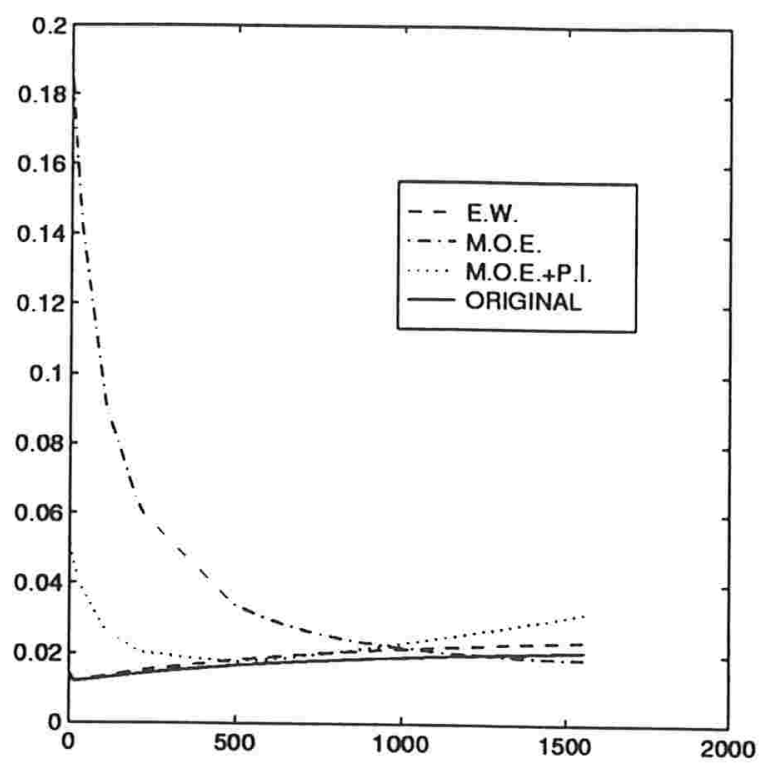

Figura 6.5. Gráficos das funções intensidades $\lambda(t)$ para os valores originais e para as estimativas de Bayes

A análise Bayesiana é similar ao caso anterior. Na Tabela 6.9 apresentamos os resumos a posteriori para $S=1000$ amostras de Gibbs.

Na tabela 6.9, apresentamos os resumos a posteriori para os parâmetros $\alpha, \beta_{1}, \beta_{2}$ e $\theta$, usando $S=1000$ amostras de Gibbs . Também incluimos $\widehat{R}$.

Para comparar as estimações de Bayes da função intensidade para cada um dos três modelos considerados $M_{1}, M_{2}$ e $M_{3}$, onde, $M_{1}=$ Modelo Weibull-exponenciada $M_{2}=$ Modelo superposição Musa e Okumoto com Exponencial considerando probabilidades de incidência

$M_{3}=$ Modelo superposição Musa e Okumoto com Exponencial , 
Calculamos estimações de Monte Carlo para $E\left(\lambda(t) \mid \mathcal{D}_{t}\right)$ para cada um dos modelos $M_{1}, M_{2}$ e $M_{3}$, baseadas nas respectivas $S=1000$ amostras de Gibbs e comparamos com os valores originais. Isto é o que apresentamos na Tabela 6.10. Os gráficos das funções intensidades são apresentados na figura 6.5.

\begin{tabular}{cccccccccc}
\hline \hline $\mathrm{t}$ & Original & $M_{3}$ & $M_{2}$ & $M_{1}$ & $\mathrm{t}$ & Original & $M_{3}$ & $M_{2}$ & $M_{1}$ \\
\hline \hline 1.268 & 0.014 & 0.013 & 0.051 & 0.187 & 831.330 & 0.018 & 0.020 & 0.021 & 0.024 \\
15.730 & 0.012 & 0.012 & 0.045 & 0.163 & 886.657 & 0.019 & 0.021 & 0.021 & 0.023 \\
32.238 & 0.012 & 0.012 & 0.040 & 0.140 & 887.428 & 0.019 & 0.021 & 0.021 & 0.023 \\
110.411 & 0.013 & 0.013 & 0.026 & 0.090 & 917.094 & 0.019 & 0.021 & 0.022 & 0.023 \\
113.406 & 0.013 & 0.013 & 0.026 & 0.089 & 921.830 & 0.019 & 0.021 & 0.022 & 0.023 \\
200.890 & 0.014 & 0.015 & 0.021 & 0.064 & 968.734 & 0.019 & 0.021 & 0.023 & 0.022 \\
221.128 & 0.014 & 0.015 & 0.020 & 0.060 & 980.527 & 0.019 & 0.0213 & 0.023 & 0.022 \\
490.283 & 0.016 & 0.018 & 0.018 & 0.034 & 1062.167 & 0.019 & 0.022 & 0.024 & 0.021 \\
499.846 & 0.017 & 0.018 & 0.018 & 0.034 & 1066.978 & 0.019 & 0.022 & 0.024 & 0.021 \\
554.878 & 0.017 & 0.019 & 0.018 & 0.031 & 1092.425 & 0.019 & 0.022 & 0.024 & 0.021 \\
565.072 & 0.017 & 0.019 & 0.018 & 0.031 & 1093.954 & 0.019 & 0.022 & 0.024 & 0.021 \\
639.517 & 0.017 & 0.019 & 0.019 & 0.029 & 1136.136 & 0.019 & 0.022 & 0.024 & 0.021 \\
656.161 & 0.017 & 0.019 & 0.019 & 0.028 & 1147.000 & 0.020 & 0.022 & 0.025 & 0.020 \\
671.523 & 0.018 & 0.019 & 0.019 & 0.028 & 1340.854 & 0.020 & 0.023 & 0.028 & 0.019 \\
719.577 & 0.018 & 0.020 & 0.019 & 0.026 & 1382.168 & 0.020 & 0.023 & 0.029 & 0.021 \\
780.015 & 0.018 & 0.020 & 0.020 & 0.025 & 1410.871 & 0.020 & 0.023 & 0.029 & 0.019 \\
811.176 & 0.018 & 0.020 & 0.020 & 0.024 & 1414.145 & 0.020 & 0.023 & 0.029 & 0.019 \\
826.838 & 0.018 & 0.020 & 0.021 & 0.024 & 1560.621 & 0.021 & 0.024 & 0.032 & 0.019 \\
\hline \hline
\end{tabular}

Tabela 6.10. Valores originais e estimativas de Monte Carlo para $\mathrm{E}(\lambda(\mathbf{t}) \mid \mathcal{D})$.

Da Tabela 6.10 e da Fig. 6.5, observamos um ajuste melhor para o modelo Weibullexponencial, desde que as estimativas de Bayes são mais próximas dos valores originais 
$\operatorname{de} \lambda(t)$.

Observamos que com Original, denotamos o modelo original.

Para fazer a seleção de modelos, estimamos a função de verossimilhança marginal para cada modelo considerado. Os valores destas estimativas são dadas na Tabela 6.11.

\begin{tabular}{cc}
\hline \hline Modelo & Verossimilhança marginal estimada \\
\hline \hline$M_{3}$ & $1.450 \cdot 10^{-80}$ \\
\hline$M_{2}$ & $9.208 \cdot 10^{-78}$ \\
\hline$M_{1}$ & $9.244 \cdot 10^{-77}$ \\
\hline \hline
\end{tabular}

Tabela 6.11. Valores estimados para a função de verossimilhança marginal.

Usando a tabela 6.11, podemos ordenar os modelos, $M_{1}, M_{2}$ e $M_{3}$ da seguinte forma,

$$
M_{3} \preceq M_{2} \preceq M_{1},
$$

e assim concluir que o melhor modelo para ajustar os dados da Tabela 6 é o modelo $M_{1}$. 


\subsection{Exemplo para o Capítulo 4}

Considerando, $\beta_{1}=20, \beta_{2}=100, \beta_{3}=0.0005, \beta_{4}=0.00000025,60$ observações foram geradas para a superposição dos processos dependentes $M_{1}(t)$ e $M_{2}(t)$ e são apresentadas na seguinte tabela:

\begin{tabular}{rccccccc}
\hline \hline$i$ & $t_{i}$ & $i$ & $t_{i}$ & $i$ & $t_{i}$ & $i$ & $t_{i}$ \\
\hline \hline 1 & 0.03188 & 16 & 78.66315 & 31 & 175.31081 & 46 & 220.67255 \\
2 & 1.86322 & 17 & 84.79987 & 32 & 182.94275 & 47 & 223.43758 \\
3 & 2.97046 & 18 & 86.17157 & 33 & 184.46696 & 48 & 226.53587 \\
4 & 9.46388 & 19 & 101.83846 & 34 & 185.78904 & 49 & 230.67443 \\
5 & 13.52815 & 20 & 109.52367 & 35 & 195.97553 & 50 & 235.61740 \\
6 & 14.68354 & 21 & 110.10556 & 36 & 197.30789 & 51 & 239.28653 \\
7 & 26.20332 & 22 & 117.50817 & 37 & 198.61468 & 52 & 241.84980 \\
8 & 27.67757 & 23 & 135.06556 & 38 & 199.36655 & 53 & 247.96334 \\
9 & 34.25170 & 24 & 141.98338 & 39 & 208.48278 & 54 & 247.97918 \\
10 & 37.84620 & 25 & 150.42468 & 40 & 209.65087 & 55 & 258.53590 \\
11 & 49.63593 & 26 & 156.60511 & 41 & 210.08397 & 56 & 258.89939 \\
12 & 55.66834 & 27 & 158.78559 & 42 & 211.20960 & 57 & 259.56239 \\
13 & 67.18403 & 28 & 163.03579 & 43 & 212.42474 & 58 & 261.01777 \\
14 & 67.27284 & 29 & 171.94196 & 44 & 212.59173 & 59 & 280.79114 \\
15 & 67.49072 & 30 & 174.77869 & 45 & 218.66697 & 60 & 291.37113 \\
\hline \hline 12 & & & & & & &
\end{tabular}

Tabela 6.12. Dados gerados para a superposição dos processos dependentes $\mathbf{M}_{1}(\mathrm{t})$ e $\mathbf{M}_{2}(\mathrm{t}) \operatorname{com} \beta_{1}=20, \beta_{2}=100, \beta_{3}=0.0005, \beta_{4}=0.00000025$.

A partir das distribuições condicionais para $I, \beta_{1}, \beta_{2}, \beta_{3}$ e,$\beta_{4}$ dadas em (4.26) até (4.29), usando o método de Gibbs com Metropolis como já foi explicado anteriormente, geramos uma amostra de tamanho 1000 para cada parâmetro. 


\begin{tabular}{ccccc}
\hline \hline & & & \multicolumn{2}{c}{ Intervalo } \\
Parâmetro & Média & D.P. & $95 \%$ Cred & $\hat{R}$ \\
\hline \hline$\beta_{1}$ & 20.13031 & 0.60938 & $(18.99845 ; 21.36982)$ & 1.002 \\
$\beta_{2}$ & 99.98655 & 1.42321 & $(97.30187 ; 102.57228)$ & 1.005 \\
$\beta_{3}$ & 0.00050696 & 0.000069888 & $(0.00038052 ; 0.00065214)$ & 1.002 \\
$\beta_{4}$ & 0.000000042262 & 0.00000013252 & $(0.00000013252 ; 0.000000029934)$ & 1.009 \\
\hline \hline
\end{tabular}

Tabela 6.13.Resumos a posteriori da superposição dos processos dependentes

$$
M_{1}(t) \text { e } M_{2}(t)
$$

Na Tabela 6.13, apresentamos resumos a posteriori dos parâmetros $\beta_{1}, \beta_{2}, \beta_{3}$ e $\beta_{4}$ usando os valores $a_{1}=1000, b_{1}=50, a_{2}=5000, b_{2}=50, a_{3}=50, b_{3}=100000, a_{4}=25$, $b_{4}=100000000$ e considerando $S=1000$ amostras de Gibbs. Também incluímos na Tabela 6.13, uma estimativa da redução de escala potencial $\hat{R}$, (veja Gelman e Rubin, 1992), para todos os parâmetros. Neste caso, o número de iterações considerado é suficiente, pois para verificar a convergência é suficiente que $\sqrt{\widehat{R}}<1.1$ para todos os parâmetros.

Na figura 6.6, apresentamos os histogramas suavizados correspondentes aos parâmetros $\beta_{1}, \beta_{2}, \beta_{3}$ e $\beta_{4}$.

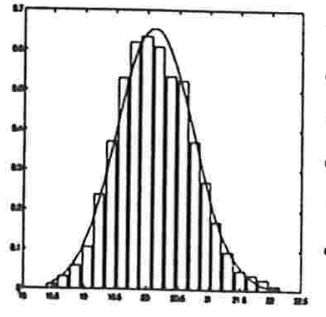

$\beta_{1}$

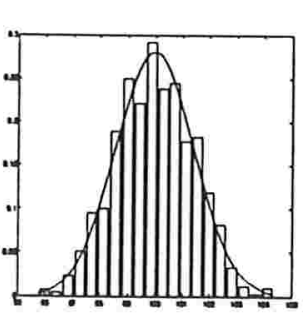

$\beta_{2}$

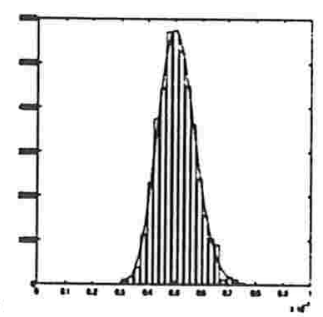

$\beta_{3}$

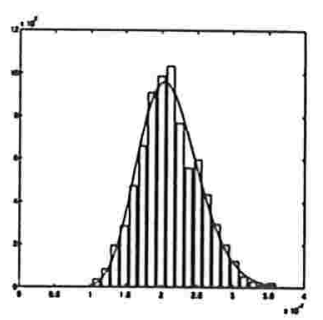

$\beta_{4}$

Figura 6.6. Densidades marginais a posteriori aproximadas 
$\mathrm{Na}$ figura 6.7, observamos os gráficos das iterações versus os valores que toma cada parâmetro nas 1000 observações, ou seja a parte estabilizada de cada cadeia do algoritmo de Gibbs com Metropolis.

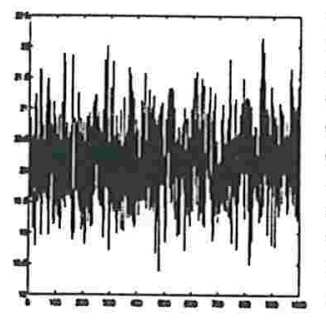

$\beta_{1}$

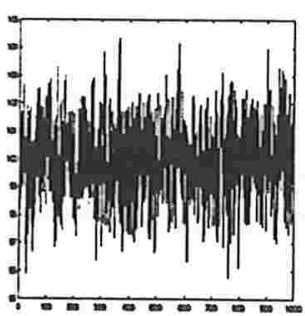

$\beta_{2}$

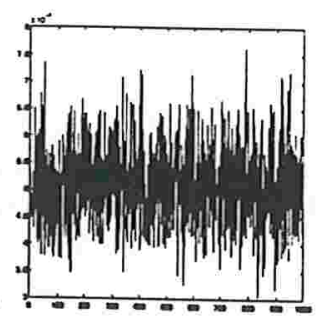

$\beta_{3}$

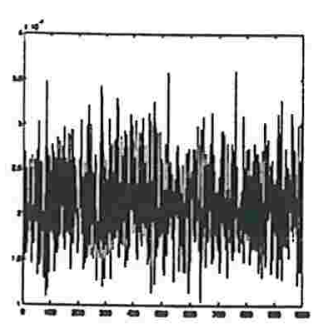

$\beta_{4}$

Figura 6.7. Gráficos das iterações

Na figura 6.8 apresentamos o gráfico da função intensidade para o caso independente (-) e dependente (- -) de superposição de processos PPNH, ou seja, em nosso exemplo quando consideramos $U(t)=0$ e $U(t)=\frac{\beta_{1}}{\beta_{2}+t}$, respectivamente. Podemos apreciar que a diferença é mais forte quando consideramos intervalos de tempo menores. Isto é claro, pois a correlação é maior quanto mais perto estivermos da origem. Na figura 6.9 são apresentados os gráficos para $\rho(t)$ real (-) e simulado por MCMC (- -). Também podemos observar nesta figura que

$$
\lim _{t \rightarrow \infty} \rho(t)=0
$$

Podemos concluir que é importante analisar a hipóteses de independência na superposição de processos PPNH, pois no caso que esta hipótese seja falsa, ocorrerá que naquelas regiões nas quais $\rho(t)$ é grande, o erro cometido não levando em conta a dependência poderá ser considerável. No exemplo analisado aqui, a região em que $\rho(t)$ é grande não pode ser deixada de lado. 


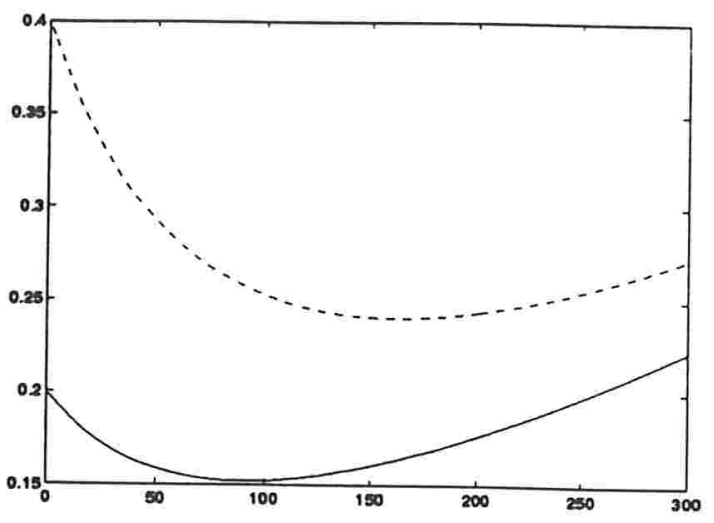

Figura 6.8. Gráficos de $\lambda(t)$

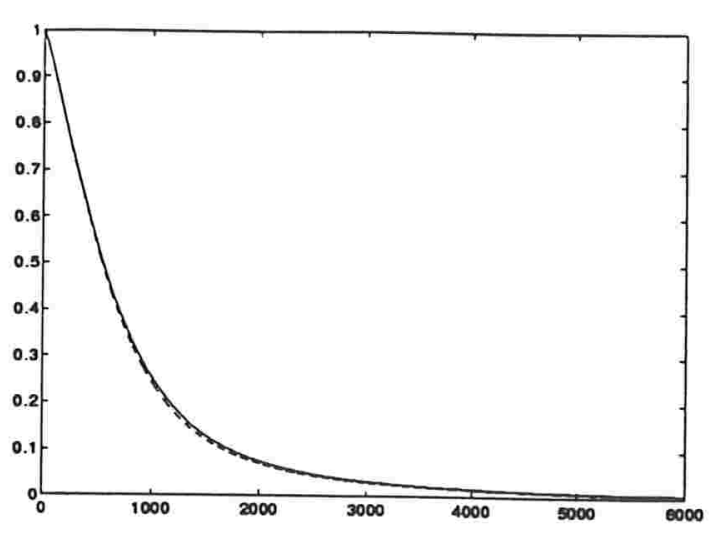

Figura 6.9. Gráficos de $\rho(t)$ 


\section{Capítulo 7}

\section{Conclusões e perspectivas futuras}

Nesta tese, mostramos que o uso de métodos Bayesianos com técnicas de simulação MCMC, (Monte Carlo em Cadeias de Markov), são apropriados para a obtenção de sumários a posteriori para os parâmetros de modelos envolvendo processos de Poisson não-homogêneos. Com o uso de técnicas Bayesianas, podemos considerar modelos mais sofisticados para analisar dados de confiabilidade de software. Novos modelos assumindo superposição de processos de Poisson não-homogêneos na presença de covariáveis foram introduzidos nesta tese.

Em geral, há informações adicionais como tamanho de software e programadores diferentes, que podem ser incorporadas no modelo como covariáveis. Esses modelos podem se ajustar melhor aos dados, conforme observamos nas ilustrações numéricas consideradas.

Também, vamos aprofundar no estudo da superposição de processos de Poisson nãohomogêneos dependentes, o que pode ser mais realístico para os dados. Esta pesquisa continuará sendo desenvolvida em conjunto com o Dr. Josemar Rodrigues.

O uso de processos de Poisson não-homogêneos com função intensidade Weibullexponenciada também pode ser de amplo interesse, dada a grande flexibilidade de ajuste.

É importante salientar que esses modelos podem ser utilizados com outros tipos de 
dados, (por exemplo, em ecologia, em medicina), e isso pode ser objetivo de pesquisas futuras. Também podemos assumir a presença de duas ou mais covariáveis, estendendo os modelos propostos nesta tese.

O trabalho computacional desenvolvido para a obtenção das quantidades a posteriori de interesse nesta tese foi feito usando o software MATLAB. 


\section{Apêndice A}

\section{Processos de Poisson}

Este resumo está baseado no texto Stochastics Processes, de Ross, 1996.

\section{A.1 Definições básicas}

1. O processo de contagem $N(t), t \geq 0$ é um processo no qual as seguintes condições são satisfeitas:

i) $N(t)$ assume somente valores inteiros não-negativos.

ii) Se $s<t$, então $N(s) \leq N(t)$.

2. Um processo $N(t), t \geq 0$ é um processo que têm incrementos independentes, se $s_{1}<t_{1}<s_{2}<t_{2}$, então $N\left(t_{1}\right)-N\left(s_{1}\right)$ é independente de $N\left(t_{2}\right)-N\left(s_{2}\right)$.

3. Um processo $N(t), t \geq 0$ é um processos que têm incrementos estacionários, se $s_{1}<t_{1}$ e $h \geq 0$, então $N\left(t_{1}+h\right)-N\left(s_{1}+h\right)$ têm a mesma distribuição que $N\left(t_{1}\right)-N\left(s_{1}\right)$.

As interpretações destas definições, em nosso contexto, são as seguintes: 
i) Num processo de contagem $N(t)$ representa o número total de falhas que ocorrem no intervalo $(0, t]$.

ii) Um processo de contagem têm incrementos independentes se o número de falhas que ocorrem em intervalos disjuntos de tempo são independentes.

iii) Um processo de contagem têm incrementos estacionários se a distribuição do número de falhas que ocorrem em qualquer intervalo de tempo depende só do comprimento do intervalo.

iv) Uma função $g$ é chamada de $o(h)$ se satisfaz:

$$
\lim _{h \rightarrow 0} \frac{g(h)}{h}=0
$$

\section{A.2 Processos de Poisson}

1. O processo de contagem $\{N(t), t \geq 0\}$ é um processo PPH com taxa de falhas $\lambda>0$, se satisfaz as seguintes propriedades:

i) $N(0)=0$.

ii) O processo têm incrementos independentes.

iii) O processo têm incrementos estacionários.

iv) $P[N(h)=1]=\lambda h+o(h)$.

v) $P[N(h) \geq 2]=o(h)$.

\section{Notação.}

Para indicar que $\{N(t), t \geq 0\}$ é um processo PPH com taxa de falhas $\lambda>0$, é suficiente escrever:

$$
N(t) \sim \operatorname{Poisson}(\lambda t)
$$




\section{Proposição.}

Seja $\{N(t), t \geq 0\}$ um processo PPH com taxa de falhas $\lambda>0$, então:

$$
P[N(h+t)-N(h)=n]=\frac{e^{-\lambda t}(\lambda t)^{n}}{n !}
$$

onde $n=0,1,2, \ldots$

Assim, a interpretação desta proposição é a seguinte: A distribuição do número de falhas em qualquer intervalo de tempo de comprimento $t$ é dado por (A.1), ou seja, depende somente de $t$.

2. O processo de contagem $\{N(t), t \geq 0\}$ é um processo PPNH com função intensidade $\lambda(t), t \geq 0$, se satisfaz as seguintes propriedades:

i) $N(0)=0$.

ii) $O$ processo têm incrementos independentes.

iii) $P[N(t+h)-N(t)=1]=\lambda(t) h+o(h)$.

iv) $P[N(t+h)-N(t) \geq 2]=o(h)$.

Definição A função de valor médio, $m(t)$, é definida por:

$$
m(t)=\int_{0}^{t} \lambda(u) d u
$$

\section{Notação.}

Para indicar que $\{N(t), t \geq 0\}$ é um processo PPNH com função intensidade $\lambda(t)>0$, escrevemos:

$$
N(t) \sim \operatorname{Poisson}(m(t))
$$

onde $m(t)$ é dada por (A.2)

\section{Proposição.}

Seja $\{N(t), t \geq 0\}$ um processo PPNH com função intensidade $\lambda(t)>0$, então: 


$$
P[N(h+t)-N(h)=n]=\frac{e^{-[m(h+t)-m(h)]}[m(h+t)-m(h)]^{n}}{n !}
$$

onde $n=0,1,2, \ldots$

Assim, a interpretação desta proposição é a seguinte: A distribuição do número de falhas no intervalo $[h, h+t)$ é dada por:

$$
N(h+t)-N(h) \sim \text { Poisson }(m(h+t)-m(h)), \quad t \geq 0
$$

ou seja, depende de t e de h. 


\section{Apêndice B}

\section{A distribuição de Hermite}

\section{B.1 Definições}

O material apresentado neste apêndice é fundamental para o desenvolvimento do Cap. 4, de superposição de processos PPNH dependentes, e está baseado no artigo, Kemp, C.D. e Kemp, A.W., 1965.

As distribuições de Poisson generalizadas são aquelas que tem função característica da seguinte forma,

$$
\phi(t)=\exp (\lambda[\psi(t)-1])
$$

onde, $\psi(t)$ também é uma função característica correspondente a uma variável aleatória que toma valores inteiros.

A F.G.P. , (função geradora de probabilidades), é da seguinte forma,

$$
\phi(s)=\exp (\lambda[f(s)-1])
$$

onde $f(s)$ também é uma F.G.P. . 
Podemos reescrever (B.1), na seguinte forma,

$$
\phi(s)=\exp \left[a_{1}(s-1)+a_{2}\left(s^{2}-1\right)+a_{3}\left(s^{3}-1\right)+\cdots\right]
$$

onde,

$$
\sum_{i=1}^{\infty} a_{i}=\lambda
$$

Se $a_{2}, a_{3}, \cdots$ são desprezíveis comparadas com $a_{1}$, a F.G.P. tende a $\phi(s)=\exp \left[a_{1}(s-1)\right]$ que é uma F.G.P. Poisson.

Se $a_{3}, a_{4}, \cdots$ são desprezíveis comparadas com $a_{1}$, então a F.G.P. tenderá a,

$$
\phi_{H}(s)=\exp \left[a_{1}(s-1)+a_{2}\left(s^{2}-1\right)\right]
$$

que é a F.G.P. da distribuição de Hermite H.

\section{B.2 Propriedades dos polinômios de Hermite, $H_{n}(x)$.}

1.

$$
\exp \left[t x-\frac{1}{2} t^{2}\right]=\sum_{n=0}^{\infty} H_{n}(x) \frac{t^{n}}{n !}
$$

2.

$$
H_{n}(x)=\sum_{j=0}^{\left[\frac{1}{2} n\right]} \frac{(-1)^{j} n ! x^{n-2 j}}{(n-2 j) ! j ! 2^{j}}
$$

3.

$$
\frac{d}{d x}\left[H_{n}(x)\right]=n H_{n-1}(x)
$$

4.

$$
H_{n+1}(x)=x H_{n}(x)-n H_{n-1}(x)
$$




\section{B.3 Propriedades dos polinômios de Hermite modificados, $H_{n}^{*}(x)$.}

1.

$$
\exp \left[t x+\frac{1}{2} t^{2}\right]=\sum_{n=0}^{\infty} H_{n}^{*}(x) \frac{t^{n}}{n !}
$$

2.

$$
H_{n}^{*}(x)=\sum_{j=0}^{\left[\frac{1}{2} n\right]} \frac{n ! x^{n-2 j}}{(n-2 j) ! j ! 2^{j}}
$$

3.

$$
\frac{d}{d x}\left[H_{n}^{*}(x)\right]=n H_{n-1}^{*}(x)
$$

4.

$$
H_{n+1}^{*}(x)=x H_{n}^{*}(x)+n H_{n-1}^{*}(x)
$$

5.

$$
H_{n}^{*}(x)=i^{-n} H_{n}(i x)
$$

B.4 Cálculo da f.d.p. da distribuição de Hermite .

Substituindo

$$
a_{1}=\alpha \beta \quad, \quad a_{2}=\frac{1}{2} \alpha^{2},
$$

em (B.2), obtemos a F.G.P. de H,

$$
\begin{array}{r}
\phi_{H}(s)=\exp \left[\alpha \beta(s-1)+\frac{1}{2} \alpha^{2}\left(s^{2}-1\right)\right] . \\
\phi_{H}(s)=\exp \left[-\left(\alpha \beta+\frac{1}{2} \alpha^{2}\right)\right] \exp \left[\alpha \beta s+\frac{1}{2} \alpha^{2} s^{2}\right] .
\end{array}
$$


Usamos (B.4) para obter,

$$
\phi_{H}(s)=\exp \left[-\left(\alpha \beta+\frac{1}{2} \alpha^{2}\right)\right] \sum_{n=0}^{\infty} H_{n}^{*}(\beta) \frac{\alpha^{n} s^{n}}{n !}
$$

Voltando às variáveis originais $a_{1}$ e $a_{2}$ de (B.5) obtemos,

$$
\begin{gathered}
\alpha \beta+\frac{1}{2} \alpha^{2}=a_{1}+a_{2}, \\
\alpha^{n}=2^{\frac{n}{2}} a_{2}^{\frac{n}{2}}, \\
H_{n}^{*}(\beta)=\sum_{j=0}^{\left[\frac{1}{2} n\right]} \frac{n ! \beta^{n-2 j}}{(n-2 j) ! j ! 2^{j}} \\
H_{n}^{*}(\beta)=\sum_{j=0}^{\left[\frac{1}{2} n\right]} \frac{n ! a_{1}^{n-2 j} a_{2}^{j-\frac{n}{2}}}{(n-2 j) ! j ! 2^{\frac{n}{2}}}
\end{gathered}
$$

Substituindo (B.6) e (B.7) em (B.5) obtemos,

$$
\phi_{H}(s)=\exp \left[-\left(a_{1}+a_{2}\right)\right] \sum_{n=0}^{\infty} \sum_{j=0}^{\left[\frac{1}{2} n\right]}\left[\frac{a_{1}^{n-2 j} a_{2}^{j}}{(n-2 j) ! j !}\right] s^{n} .
$$

Logo, a f.d.p. da distribuição de uma variável Hermite , H, é dada na seguinte forma,

$$
P[H=h]=\exp \left[-\left(a_{1}+a_{2}\right)\right] \sum_{n=0}^{\infty} \sum_{j=0}^{\left[\frac{1}{2} h\right]}\left[\frac{a_{1}^{h-2 j} a_{2}^{j}}{(h-2 j) ! j !}\right] .
$$




\section{Apêndice $\mathrm{C}$}

\section{A distribuição de Poisson Bivariada}

Uma das formas de construir a distribuição de Poisson Bivariada é o chamado Método de Redução Trivariado, ( veja, Kocherlakota,S. e Kocherlakota,K., 1992 ). Sejam $W_{1}, W_{2}$ e $W_{3}$ tres v.a.i. com distribuições de Poisson com parâmetros $\lambda_{1}, \lambda_{2}$ e $\lambda_{3}$, respectivamente. Sejam também as variáveis $X$ e $Y$, dadas por:

$$
X=W_{1}+W_{3} \quad \text { e } \quad Y=W_{2}+W_{3} .
$$

Então, a F.G.P. (função geradora de probabilidades) de (X,Y) é da seguinte forma:

$$
\phi_{(X, Y)}\left(t_{1}, t_{2}\right)=E\left[t_{1}^{X} t_{2}^{Y}\right]=E\left[t_{1}^{W_{1}} t_{2}^{W_{2}}\left(t_{1} t_{2}\right)^{W_{3}}\right],
$$

ou seja,

$$
\phi_{(X, Y)}\left(t_{1}, t_{2}\right)=\exp \left[\lambda_{1}^{*}\left(t_{1}-1\right)+\lambda_{2}^{*}\left(t_{2}-1\right)+\lambda_{3}\left(t_{1}-1\right)\left(t_{2}-1\right)\right],
$$

onde, $\lambda_{1}^{*}=\lambda_{1}+\lambda_{3} \quad e \quad \lambda_{2}^{*}=\lambda_{2}+\lambda_{3}$.

Também temos que,

$$
\begin{array}{r}
\operatorname{Cov}(X, Y)=\operatorname{Cov}\left(W_{1}+W_{3}, W_{2}+W_{3}\right)=\operatorname{Var}\left(W_{3}\right)=\lambda_{3} . \\
\operatorname{Var}(X)=\operatorname{Var}\left(W_{1}+W_{3}\right)=\lambda_{1}+\lambda_{3} .
\end{array}
$$




$$
\begin{gathered}
\operatorname{Var}(Y)=\operatorname{Var}\left(W_{2}+W_{3}\right)=\lambda_{2}+\lambda_{3} . \\
\rho(X, Y)=\frac{\lambda_{3}}{\left[\left(\lambda_{1}+\lambda_{3}\right)\left(\lambda_{2}+\lambda_{3}\right)\right]^{\frac{1}{2}}} .
\end{gathered}
$$

Assim, neste modelo $\rho(X, Y) \geq 0$ pois $\lambda_{3} \geq 0$. 


\section{Apêndice D}

\section{Geração de processos de Poisson}

Este resumo está baseado no texto A Course in Simulation de Ross, 1991, e no artigo de Lewis e Shedler, 1979.

\section{Processos PPH}

Consideremos um processo PPH com taxa de falhas $\lambda>0$, e o seguinte resultado: Proposição

Os tempos entre falhas sucessivas são v.a.i.i.d., $T_{i}$, com distribuição $\operatorname{Exp}(\lambda)$.

Assim, esses tempos entre falhas $T_{1}, T_{2}, \cdots, T_{n}$ podem ser obtidos gerando $n$ v.a.i.i.d. uniformes em $(0,1), U_{1}, U_{2}, \cdots, U_{n}$ tais que:

$$
T_{i}=-\frac{\log \left(U_{i}\right)}{\lambda}
$$

onde $i=1,2, \cdots, n$.

Algoritmo para a geração de tempos entre falhas de um processo PPH com taxa de falhas $\lambda$ num intervalo de tempo $(0, T]$.

$t=0 ; I=0 ; T>0$ dado; $\lambda>0$ dado;

enquanto $t \leq T$

- Gerar um número aleatório $U$ uniforme em $[0,1]$ 
Gerar um número aleatório $U$ uniforme em $[0,1]$

Calcular $t=t-\frac{\log U}{\lambda}$

$I=I+1 ; \quad S(I)=t$

- fim.

Notemos que $S(I)$ representa o último tempo gerado em $(0, t]$ e é tal que:

$S(1)<S(2)<\cdots<S(I-1)<S(I) \leq t$, e $I$ representa o número de falhas em $(0, t]$.

\section{Processos PPNH}

Seja um processo PPNH com função intensidade $\lambda(t) \geq 0$.

O método que consideramos é chamado de Thinning Method, (veja, Lewis e Shedler, 1979). Começamos escolhendo um valor $\lambda>0$ tal que seja uma limitante da função intensidade, ou seja

$$
\lambda(t) \leq \lambda \quad \forall t \in(0, t]
$$

onde $(0, t]$ é o intervalo no qual queremos gerar os tempos de falhas . Este método está baseado no seguinte resultado:

\section{Proposição}

Suponhamos que as falhas ocorrem segundo um processo PPH com taxa de falhas $\lambda$ e, independentemente de ocorrências anteriores, uma falha que ocorre no tempo $t$ é escolhida com probabilidade $p_{t}$. Então, o processo formado por estes tempos escolhidos é um processo PPNH com função intensidade

$$
\lambda(t)=\lambda p_{t}
$$

\section{Algoritmo para a geração de tempos de um processo PPNH} com função intensidade $\lambda(t)$ num intervalo de tempo $(0, T]$.

$$
t=0 ; I=0 ; T>0 \text { dado; } \lambda>0 \text { dado; }
$$


- enquanto $t \leq T$

Gerar um número aleatório $U_{1}$ uniforme em $[0,1]$

Calcular $t=t-\frac{\log U_{1}}{\lambda}$.

Calcular $\lambda(t)$.

gerar um número aleatório $U_{2}$ uniforme em $[0,1]$

Se $U_{2} \leq \frac{\lambda(t)}{\lambda}$ fazer $I=I+1 ; \quad S(I)=t ;$

- fim.

Em algumas situações é preciso dispor de um método mas eficiente, o qual é conseguido, particionando o nosso intervalo, $(0, t]$, no qual pretendemos gerar os tempos de falhas . Assim, obtemos,

$$
(0, t]=\left(0, t_{1}\right] \cup\left(t_{1}, t_{2}\right] \cup \cdots \cup\left(t_{n-1}, T\right)
$$

e escolhemos limitantes para cada sub-intervalo. Assim, se $\lambda_{1}, \cdots, \lambda_{n}$ são essas limitantes então,

$$
\lambda(t) \leq \lambda_{i} \quad \text { se } \quad t \in\left(t_{i-1}, t_{i}\right] \quad \text { com } \quad i=1, \cdots, n
$$

onde $t_{0}=0$ e $t_{n}=T$.

Algoritmo modificado para a geração de tempos de um processo PPNH com função intensidade $\lambda(t)$ num intervalo de tempo $(0, T]$ particionado em $n$ sub-intervalos de acordo com (??) .

$t=0 ; I=0 ; T_{j}>0$ dado; $\lambda_{j}>0$ dado; $k>0$ inteiro dado;

Gerar um número aleatório $U_{1}$ uniforme em $[0,1]$;

Calcular $x=-\frac{\log U_{1}}{\lambda_{1}}$; 
- Fazer $\mathrm{j}=1: \mathrm{k}$

enquanto $t+x \leq T_{j}$

Fazer $t=t+x$

Calcular $\lambda(t)$

Gerar um número aleatório $U_{2}$ uniforme em $[0,1]$

Se $U_{2} \leq \frac{\lambda(t)}{\lambda_{j}}$ fazer $I=I+1 ; \quad S(I)=t ; \quad L(I)=\lambda(t) ;$

Gerar um número aleatório $U_{3}$ uniforme em $[0,1]$

Calcular $x=-\frac{\log U_{3}}{\lambda_{j}}$.

fim.

- Se $j<k$ fazer $x=\frac{\left(x-T_{j}+t\right) \lambda_{j}}{\lambda_{j+1}} ; \quad t=T_{j}$;

- fim. 


\section{Referências Bibliográficas}

[1] Achcar, J.A. (1995). Use of Approximate Bayesian Inference for Software Reliability. Notas do ICMSC-USP, 18. São Carlos, S.P. Brasil.

[2] Achcar, J.A., Dey,D. e Niverthy,M. (1998). A Bayesian Approach using nonhomogeneous Poisson process for software reliability models in Frontiers in Reliability. Series on Quality, Reliability and Engineering Statistics-Vol.4. S.K. Basu. and S. Mukhopadhyay, eds., Calcuta University. India.

[3] Aslanidou, H., Dey,D. e Sinha,D. (1998). Bayesian analysis of multivariate survival data using Monte Carlo methods. The Canadian J. of Statistics, La revue Canadienne de Statistique, 26, 1, 33-48.

[4] Basawa, I.V., and Prakasa Rao, B.L.S. (1980). Statistical Inference for Stocastics Processes. London: Academic Press.

[5] Campodonico, S., e Singpurwalla, N.D. (1995). Inference and predictions from Poisson point processes incorporating expert knowledge. J. Amer. Statist. Assoc., 90, 429, 220-226.

[6] Cancho, V.G., Bolfarine, H., e Achcar, J.A. (1999). A Bayesian Analysis for the Exponentiated-Weibull distribution. Journal of Applied Statistical Science, 8, 4, 227-242.

[7] Carlin, B.P. and Louis, T.A. (1996). Bayes and empirical Bayes methods for data analysis. New York: Chapman and Hall. 
[8] Casella, G. and George, E.I. (1992). Explaining the Gibbs Sampler. Amer. Statistician, 46, 167-174.

[9] Chib, S. and Greenberg, E. (1995). Understanding the Metropolis-Hasting algorithm. The American Statistician, 49, 327-335.

[10] Cox, D.R., and Lewis, P.A. (1966). Statistical Analysis of Series of Events. London. Methuen.

[11] Duane,J.T.(1966). IEEE Transactions on Aerospace, AS-2(2), 563-566.

[12] Gaver, D.P. and Acar, M. (1979). Analytical hazard representations for use in reliability, mortality and simulation studies. Comm. in Statist.,Sim. and Com. B8, 91-111.

[13] Gelfand, A.E. and Smith, A.F.M. (1990). Sampling-based approaches to calculating marginal densities. J. Amer. Statist. Assoc., 85, 398-409.

[14] Gelman, A., Carlin J.B., Stern H.S. and Rubin, D.B. (1995). Bayesian Data Analysis. New York: Chapman and Hall.

[15] Gelman, A., and Rubin, D.B. (1992). Inference from iterative simulation using multiple sequences (with discussion). Statistical Science, 7, 457-511.

[16] Goel, A.L., and Okumoto, K. (1979). Time dependent error detection rate model for software reliability and other performance measures, IEEE Transactions on Reliability, 28, 206-211.

[17] Jelinsky,Z. and Moranda, P.B. (1972). Prediction of Software Reliability Research. In Statistical Computer Performance Evaluation, W.Freiberger, ed., New York: Academic Press, pp. 465-497.

[18] Kemp, C.D. and Kemp, A.W. (1965). Some properties of the "Hermite"distribuição . Biometrika, 52,3-4, 763-773. 
[19] Kocherlakota,S. and Kocherlakota,K. (1992). Bivariate discrete distribuições . London: Marcel Deckker, Inc.

[20] Kuo, L. and Yang, T.Y. (1996). Bayesian computation for nonhomogeneous Poisson process in software reliability. J. Amer. Statist. Assoc., 91, 763-773.

[21] Langberg, N. e Singpurwalla, N.D. (1985). A Unification of some Software Reliability Models. SIAM, Journal on Scientific and Statistical Computing, 6, 781-790.

[22] Lewis, P.A.W. and Shedler, G.S. (1979). Simulation of Nonhomogeneous Poisson Process by Thinning. Naval Research Logistic Quarterly, 26, 403-413.

[23] Littlewood, B. and Verral, J.L. (1973). A Bayesian Reliability Growth Model for Computer Software. Applied Statistics, 22, 332-346.

[24] Mazzuchi, T.A., and Soyer, R. (1988). A Bayes Empirical-Bayes model for software reliability, IEEE Transactions on Reliability, R37:2, 248-258.

[25] Metropolis, N., Rosenbluth, A.W., Rosenbluth, M. N., Teller, A.H., and Teller, E. (1953). Equations of state calculations by fast computing machines. J. Chemical Physics, 21, 1087-1092.

[26] Miller, D.R. (1986). Exponential order statistics models of software reliability. IEEE Transaction on Software Engineering, SE-12, 12-24.

[27] Moranda, P.B. (1975). Prediction of Software Reliability and its Applications. Proceedings of the Annual Reliability and Maintainability Symposium. Washington,D.C., pp. 327-332.

[28] Mudholkar, G.S., Srivastava, D.K. and Friemer,M.(1982). The exponentiated-Weibull family: a reanalysis of the bus-motor failure data. Technometrics, $37,436-445$.

[29] Musa, J.D.,Iannino, A. and Okumoto,K.(1987). Software reliability: measurement, prediction, aplication. New York:Mc.Graw Hill. 
[30] Musa, J.D. and Okumoto K. (1984). A logarithmic Poisson execution time model for software reliability measurement. Proceedings Seventh International Conference on Software Engineering. Orlando, pp. 230-238.

[31] Raftery, A.E.(1996). Hypothesis testing and model selection. In Markov Chain Monte Carlo in practice, W.Gilks, S.Richardson and D.J. Spiegelhalter, eds., London: Chapman and Hall, pp. 163-187.

[32] Ross, S.M.(1991). A Course in Simulation. New York: Macmillan Publishing Co.

[33] Ross, S.M.(1996). Stochastics Processes. New York: John Wiley and Sons.

[34] Smith, A.F.M. and Roberts, G.O. (1993). Bayesian computation via the Gibbs sampler and related Markov Chain Monte Carlo methods. J. Roy. Statist. Soc., Ser.B, 55, 3-23.

[35] Tanner, M. and Wong, W. (1987). The calculation of posterior distributions by data augmentation. J. Amer. Statist. Assoc., 82, 528-550.

[36] Titterington, D.M., Smith, A.F.M. and Makov, U.E. (1985). Statistical Analysis of Finite Mixture Distributions. New York: John Wiley and Sons.

[37] Yamada, S. and Osaki, S. (1984). Nonhomogeneous error detection rate models for software reliability growth. Reliability Theory. Reliability Theory, eds. Osaki,S. and Hatoyama, Y. Berlin: Springer-Verlag, pp.120-143.

[38] Yamada, S. and Osaki, S. (1985). Software reliability modeling: Models and applications. IEEE Transaction on Software Engineering, 12, 1431-1437.

[39] Yang, T.Y.(1994). Computational approaches to Bayesian inference for software reliability, Ph.D. thesis, Department of Statistics, University of Connecticut, Storrs, U.S.A. 